\title{
MODULI OF NON-STANDARD NIKULIN SURFACES IN LOW GENUS
}

\author{
ANDREAS LEOPOLD KNUTSEN, MARGHERITA LELLI-CHIESA, AND ALESSANDRO VERRA
}

\begin{abstract}
Primitively polarized genus $g$ Nikulin surfaces $(S, M, H)$ are of two types, that we call standard and non-standard depending on whether the lattice embedding $\mathbb{Z}[H] \oplus_{\perp} \mathbf{N} \subset \operatorname{Pic} S$ is primitive. Here $H$ is the genus $g$ polarization and $\mathbf{N}$ is the Nikulin lattice. We concentrate on the non-standard case, which only occurs in odd genus. In particular, we study the birational geometry of the moduli space of nonstandard Nikulin surfaces of genus $g$ and prove its rationality for $g=7,11$ and the existence of a rational double cover of it when $g=9$. Furthermore, if $(S, M, H)$ is general in the above moduli space and $\left(C,\left.M\right|_{C}\right)$ is a general Prym curve in $|H|$, we determine the dimension of the family of non-standard Nikulin surfaces of genus $g$ containing $\left(C,\left.M\right|_{C}\right)$ for $3 \leq g \leq 11$; this completes the study of the Prym-Nikulin map initiated in [KLV].
\end{abstract}

\section{INTRODUCTION}

A Nikulin surface is a $K 3$ surface endowed with a non-trivial double cover branched along eight disjoint rational curves. Nikulin surfaces have attracted a lot of attention in recent time because of their relevance in the study of both the moduli [FV] and the syzygies [FK1, FK2] of Prym canonical curves. It is imperative to recall the lattice theoretical proof by Sarti and van Geemen [VGS] of the existence of exactly two types of polarized Nikulin surfaces, that we call standard and non-standard (cf. 92), the latter occurring only in odd genera. There are coarse moduli spaces $\mathcal{F}_{g}^{\mathbf{N}, s}$ and $\mathcal{F}_{g}^{\mathbf{N}, n s}$ parametrizing genus $g$ primitively polarized Nikulin surfaces of standard and non-standard type, respectively; more precisely, a point of $\mathcal{F}_{g}^{\mathbf{N}, s}$ (resp., $\left.\mathcal{F}_{g}^{\mathbf{N}, n s}\right)$ represents a triple $(S, M, H)$, where $S$ is a standard (resp., non-standard) Nikulin surface, $H \in \operatorname{Pic} S$ is a genus $g$ primitive polarization and the line bundle $M \in \operatorname{Pic} S$ defines the double cover branched along eight disjoint rational curves. Both $\mathcal{F}_{g}^{\mathbf{N}, s}$ and $\mathcal{F}_{g}^{\mathbf{N}, n s}$ are irreducible of dimension 11, cf. [Do, §3], vGS, Prop. 2.3].

Up to now, only the moduli spaces $\mathcal{F}_{g}^{\mathbf{N}, s}$ have been extensively studied, while nonstandard Nikulin surfaces have not been adequately considered. This paper aims to (partially) fill this gap. We concentrate on the $\mathbb{P}^{g}$-bundle over $\mathcal{F}_{g}^{\mathbf{N}, n s}$ parametrizing pairs $((S, M, H), C)$ such that $(S, M, H) \in \mathcal{F}_{g}^{\mathbf{N}, n s}$ and $C \in|H|$. Let $\mathcal{P}_{g}^{\mathbf{N}, n s}$ be the open set of pairs such that $C$ is smooth and let $\mathcal{R}_{g}$ be the moduli space of Prym curves; we look at the diagram

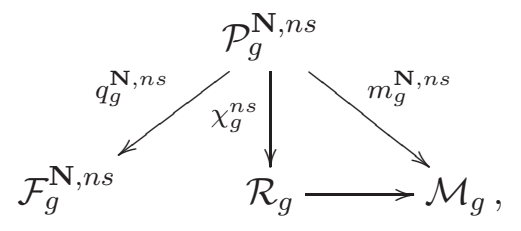


whose arrows can be described as follows: $q_{g}^{\mathbf{N}, n s}$ and $m_{g}^{\mathbf{N}, n s}$ are the obvious forgetful maps. Moreover, the Prym-Nikulin map $\chi_{g}^{n s}$ sends $((S, M, H), C)$ to the Prym curve $\left(C, M \otimes \mathcal{O}_{C}\right)$. In particular, $m_{g}^{\mathbf{N}, n s}$ is just the composition of $\chi_{g}^{n s}$ and the forgetful map $\mathcal{R}_{g} \rightarrow \mathcal{M}_{g}$

The main difference between the standard and non-standard case is that a general hyperplane section of a general polarized Nikulin surface of standard type is Brill-Noether general, while curves lying on non-standard Nikulin surfaces carry two unexpected thetacharacteristics (cf. Prop. 2.3) that make them special in moduli. A first consequence is that the maps $m_{g}^{\mathbf{N}, n s}$ and $\chi_{g}^{n s}$ can never be dominant. Furthermore, a heuristic count suggests that they cannot be generically finite for $g \leq 11$, cf. Remark 2.11, In [KLV] we proved that the map $\chi_{g}^{n s}$ is birational onto its image for (odd) genus $g \geq 13$, and the behaviour of the analogous map in the standard case was completely described. In this paper, we complete the picture by showing that:

Theorem 1.1. The map $\chi_{g}^{\text {ns }}$ has generically

- 9-dimensional fibers for $g=3$;

- 6-dimensional fibers for $g=5$;

- 4-dimensional fibers for $g=7$;

- 2-dimensional fibers for $g=9$;

- 1-dimensional fibers for $g=11$.

As already mentioned, hyperplane sections of non-standard Nikulin surfaces have some peculiar and compelling properties, that we now describe in more detail. A general genus $g$ polarized non-standard Nikulin surface $(S, M, H)$ carries two line bundles $R, R^{\prime}$ such that $H(-M) \simeq R \otimes R^{\prime}$. The restrictions of $R$ and $R^{\prime}$ to a general hyperplane section $C \in|H|$ are two theta-characteristics with positive dimensional spaces of global sections. For (odd) genus $g \geq 5$ both $h^{0}\left(\mathcal{O}_{C}(R)\right) \geq 2$ and $h^{0}\left(\mathcal{O}_{C}\left(R^{\prime}\right)\right) \geq 2$ and hence the theta divisor of the Jacobian of $C$ has two singular points of given multiplicity. We precisely describe the images of $m_{g}^{\mathbf{N}, n s}$ for $g=3$ and 5 , cf. Theorems 2.9 and 2.10.

- the image of $m_{3}^{\mathbf{N}, n s}$ is the hyperelliptic locus in $\mathcal{M}_{3}$;

- the image of $m_{5}^{\mathbf{N}, n s}$ coincides with the locus of curves in $\mathcal{M}_{5}$ possessing two autoresidual $g_{4}^{1}$; in particular, this locus is irreducible.

For $g \geq 7$ the situation becomes more intricate and the birational geometry of the moduli space $\mathcal{F}_{g}^{\mathbf{N}, n s}$ is worth investigating. We prove:

Theorem 1.2. The moduli space $\mathcal{F}_{g}^{\mathbf{N}, n s}$ of non-standard Nikulin surfaces of genus $g$ is:

- rational for $g=7$ and $g=11$;

- unirational with a rational double cover for $g=9$.

The proof of both Theorems 1.2 and 1.1 for $g \geq 7$ is given in 935 and relies on the description of nice projective models of non-standard Nikulin surfaces $(S, M, H)$ in low genus. Set $r:=h^{0}(R)-1$ and $r^{\prime}:=h^{0}\left(R^{\prime}\right)-1$. As already remarked by Garbagnati and Sarti in [GS], the line bundles $R$ and $R^{\prime}$ enable to realize $S$ as a subvariety of the intersection of the Segre variety $\mathbb{P}^{r^{\prime}} \times \mathbb{P}^{r} \subset \mathbb{P}^{r r^{\prime}+r+r^{\prime}}$ with a linear space of dimension $g-2$, namely, $\mathbb{P}\left(H^{0}(S, H(-M))^{\vee}\right)$. We are able to detect some geometric conditions that are also sufficient for such a subvariety of $\left(\mathbb{P}^{r^{\prime}} \times \mathbb{P}^{r}\right) \cap \mathbb{P}^{g-2}$ to be a Nikulin surface of non-standard type. 
For instance, a general non-standard Nikulin surface of genus 7 is a divisor of bidegree $(2,3)$ in $\mathbb{P}^{1} \times \mathbb{P}^{2}$, cf. [GS, $\left.\S 4.8\right]$. Furthermore, a $K 3$ surface in $\left|\mathcal{O}_{\mathbb{P}^{1} \times \mathbb{P}^{2}}(2,3)\right|$ is a Nikulin surface of non-standard type if and only if it contains two conics $A_{1}$ and $A_{2}$ that are contracted by the first projection $\mathbb{P}^{1} \times \mathbb{P}^{2} \rightarrow \mathbb{P}^{1}$ and are mapped to the same plane conic by the second projection $\mathbb{P}^{1} \times \mathbb{P}^{2} \rightarrow \mathbb{P}^{2}$.

Analogously, a general surface in $\mathcal{F}_{9}^{\mathbf{N}, n s}$ is a quadratic section of a Del Pezzo threefold $T:=\left(\mathbb{P}^{2} \times \mathbb{P}^{2}\right) \cap \mathbb{P}^{7} \subset \mathbb{P}^{8}$, cf. [GS, $\left.\S 4.9\right]$. Moreover, an element in $\left|\mathcal{O}_{T}(2,2)\right|$ is a nonstandard Nikulin surface if and only if it contains two sets of four lines that are contracted by the first and second projection, respectively.

As regards genus 11, a general surface $S$ in $\mathcal{F}_{11}^{\mathbf{N}, n s}$ defines a divisor of type $(1,2)$ in the threefold $T^{\prime}:=\left(\mathbb{P}^{2} \times \mathbb{P}^{3}\right) \cap \mathbb{P}^{9} \subset \mathbb{P}^{11}$. The projection $T^{\prime} \rightarrow \mathbb{P}^{3}$ realizes $T^{\prime}$ as the blow-up of $\mathbb{P}^{3}$ along a rational normal cubic curve $\gamma$ and we denote by $P_{\gamma}$ the exceptional divisor. The surface $S$ intersects $P_{\gamma}$ along a rational quintic curve $\Gamma \subset T^{\prime} \subset \mathbb{P}^{9}$ and in fact we show that the containment of $\Gamma$ is a necessary and sufficient condition for a surface in $\left|\mathcal{O}_{T^{\prime}}(1,2)\right|$ to be a non-standard Nikulin surface of genus 11 . The rationality results in Theorem 1.2 will follow from these characterizations.

Concerning the fibers of the moduli map $\chi_{g}^{n s}$, the case of genus 7 has some special features. Let $C \subset\left(\mathbb{P}^{r} \times \mathbb{P}^{r^{\prime}}\right) \cap \mathbb{P}^{g-2}$ be a general genus $g$ Nikulin section in the nonstandard case. In genus 9 a general quadratic section of the threefold $T$ containing $C$ is a non-standard Nikulin surface; the same holds in genus 11 if one considers in the threefold $T^{\prime}$ a general divisor of type $(1,2)$ through $C$. The situation in genus 7 is divergent: a general $K 3$ surface in the linear system $\left|\mathcal{I}_{C / \mathbb{P}^{1} \times \mathbb{P}^{2}}(2,3)\right|$ is not a Nikulin surface. This difference depends on the fact that, contrary to what happens for $g=9,11$, in genus 7 the embedded curve $C \subset \mathbb{P}^{g-2}$ is not quadratically normal. As a relevant consequence, the image of $\chi_{7}^{n s}$ lies in the ramification locus of the Prym map $\mathcal{R}_{7} \rightarrow \mathcal{A}_{6}$, cf. Remark 3.5 and $[\mathrm{Be}$. This suggests an interesting behaviour of Nikulin sections with respect to their Prym varieties. In the standard case this phenomenon was already pointed out in [FV], where the image of $\chi_{6}^{\mathbf{N}, s}$ is identified with the ramification locus of the Prym map $\mathcal{R}_{6} \rightarrow \mathcal{A}_{5}$, but was still unknown in the non-standard case.

Acknowledgements. The first author has been partially supported by grant n. 261756 of the Research Council of Norway. The second and third named authors were supported by the Italian PRIN-2015 project "Geometry of Algebraic varieties" and the third by GNSAGA.

\section{NikUlin surfaces of NON-STANDARD type AND SEgre Varieties}

We recall some basic definitions and properties.

Definition 2.1. A polarized Nikulin surface of genus $g \geq 2$ is a triple $(S, M, H)$ such that $S$ is a smooth $K 3$ surface, $\mathcal{O}_{S}(M), H \in \operatorname{Pic} S$ and the following conditions are satisfied:

- $S$ contains 8 mutually disjoint rational curves $N_{1}, \ldots, N_{8}$ such that

$$
N_{1}+\cdots+N_{8} \sim 2 M .
$$

- $H$ is nef, $H^{2}=2(g-1)$ and $H \cdot M=0$.

We say that $(S, M, H)$ is primitively polarized if in addition $H$ is primitive in Pic $S$. 
Definition 2.2. Let $(S, M, H)$ be a Nikulin surface of genus $g$. Its Nikulin lattice $\mathbf{N}=\mathbf{N}(S, M)$ is the rank 8 sublattice of Pic $S$ generated by $N_{1}, \ldots, N_{8}$ and $M$.

One also defines the rank 9 lattice

$$
\Lambda=\Lambda(S, M, H):=\mathbb{Z}[H] \oplus_{\perp} \mathbf{N} \subset \operatorname{Pic} S .
$$

If the embedding $\Lambda \subset \operatorname{Pic} S$ is primitive, we call $(S, M, H)$ a Nikulin surface of standard type, else we call it a Nikulin surface of non-standard type.

There are coarse moduli spaces $\mathcal{F}_{g}^{\mathbf{N}, s}$ (respectively, $\mathcal{F}_{g}^{\mathbf{N}, n s}$ ) parametrizing polarized Nikulin surfaces of genus $g$ of standard (resp., non-standard) type. Both $\mathcal{F}_{g}^{\mathbf{N}, s}$ and $\mathcal{F}_{g}^{\mathbf{N}, n s}$ are irreducible of dimension 11 and their very general members have Picard number nine, cf. [Do, §3], vGS, Prop. 2.3]. By [vGS, Prop. 2.2], if $(S, M, H)$ is a nonstandard Nikulin surface of genus $g$, then $g$ is odd and the embedding $\Lambda \subset \operatorname{Pic} S$ has index two. More precisely (cf. GS, Prop. 2.1 and Cor. 2.1]), possibly after renumbering the curves $N_{i}$, there are $R, R^{\prime} \in \operatorname{Pic} S$ such that

- $H-N_{1}-N_{2}-N_{3}-N_{4} \sim 2 R$ and $H-N_{5}-N_{6}-N_{7}-N_{8} \sim 2 R^{\prime}$ if $g \equiv 1 \bmod 4$;

- $H-N_{1}-N_{2} \sim 2 R$ and $H-N_{3}-\cdots-N_{8} \sim 2 R^{\prime}$ if $g \equiv 3 \bmod 4$.

Moreover, when $\operatorname{rk} \operatorname{Pic} S=9$, then Pic $S \simeq \mathbb{Z}[R] \oplus \mathbf{N}$ by [GS, Prop. 2.1 and Cor. 2.1]. We also need to define the line bundle $L:=H-M$, which satisfies $L^{2}=2(g-3)$ and $L \cdot N_{i}=1$ for $i=1, \ldots, 8$.

We henceforth concentrate on Nikulin surfaces of non-standard type.

First of all we show that hyperplane sections of non-standard Nikulin surfaces are rather special.

Proposition 2.3. Let $(S, M, H)$ be a general non-standard Nikulin surface of genus $g \equiv 1 \bmod 4$ (respectively, $g \equiv 3 \bmod 4$ ) and let $L, R$ and $R^{\prime}$ be as above. Then

(i) $R$ and $R^{\prime}$ are globally generated with $h^{1}(R)=h^{1}\left(R^{\prime}\right)=0$ if $g \geq 5$;

(ii) $h^{0}(L)=g-1$ and $L$ is very ample if $g \geq 7$ and is ample and globally generated defining a degree two morphism onto $\mathbb{P}^{1} \times \mathbb{P}^{1} \subset \mathbb{P}^{3}$ if $g=5$;

(iii) if $g \geq 5$, then for any smooth curve $C$ in $|H|$, the line bundles $\mathcal{O}_{C}(R)$ and $\mathcal{O}_{C}\left(R^{\prime}\right)$ are theta-characteristics satisfying $h^{0}(S, R)=h^{0}\left(C, \mathcal{O}_{C}(R)\right)=(g+3) / 4$ (resp., $(g+5) / 4)$ and $h^{0}\left(S, R^{\prime}\right)=h^{0}\left(C, \mathcal{O}_{C}\left(R^{\prime}\right)\right)=(g+3) / 4$ (resp., $\left.(g+1) / 4\right)$.

Proof. Since all properties are open in the moduli space, one may prove them for a non-standard Nikulin surface with rk Pic $S=9$. Then (i) is proved in [GS, Prop. 3.5(2)] (recalling that a linear system on a $K 3$ surface without base components is base point free) and (ii) in GS, Prop. 3.2 and Lemma 3.1], using the classical numerical criteria of Saint-Donat [SD]. As $L^{2}=2(g-3)>0$, we have $h^{1}(L)=h^{2}(L)=0$, whence $h^{0}(L)=g-1$ by Riemann-Roch.

To prove (iii) we note that $R-H \sim-\left(R+N_{1}+N_{2}+N_{3}+N_{4}\right)$ (resp., $-(R+$ $\left.N_{1}+N_{2}\right)$ ). Thus, $h^{0}(R-H)=0$. Moreover, the linear system $|R|$ contains irreducible members thanks to (i), and hence $|H-R|$ contains a divisor $D$ that is the union of an irreducible element in $|R|$ and four rational irreducible tails. In particular, one has $h^{0}\left(\mathcal{O}_{D}\right)=1$ and thus $h^{1}(R-H)=0$. The standard restriction sequence yields $h^{0}(S, R)=h^{0}\left(C, \mathcal{O}_{C}(R)\right)=\frac{1}{2} R^{2}+2$ by Riemann-Roch and (i). The rest then follows from an easy computation and the same argument applies to $R^{\prime}$. 
Remark 2.4. In the embedding $S \subset \mathbb{P}^{g-2}$ defined by $|L|$, any smooth $C$ in $|H|$ is mapped to a Prym-canonical curve, as $\left.L\right|_{C} \simeq \omega_{C} \otimes \mathcal{O}_{C}(M)$ satisfies $\left.L\right|_{C} ^{\otimes 2} \simeq \omega_{C}^{\otimes 2}$, and all $N_{1}, \ldots, N_{8}$ are mapped to lines.

From now on, we will set $r:=h^{0}(S, R)-1$ and $r^{\prime}:=h^{0}\left(S, R^{\prime}\right)-1$. By Proposition 2.3. as soon as $g \geq 7$, the two linear systems $\left|R^{\prime}\right|$ and $|R|$ (and their restrictions to $C$ ) define an embedding

$$
C \subset S \subset \mathbb{P}^{r^{\prime}} \times \mathbb{P}^{r} \subset \mathbb{P}^{r r^{\prime}+r+r^{\prime}},
$$

where the second inclusion is the Segre embedding.

Notation 2.5. We let $p: \mathbb{P}^{r^{\prime}} \times \mathbb{P}^{r} \rightarrow \mathbb{P}^{r}$ and $p^{\prime}: \mathbb{P}^{r^{\prime}} \times \mathbb{P}^{r} \rightarrow \mathbb{P}^{r^{\prime}}$ be the two projection maps. For any subvariety $X \subset \mathbb{P}^{r^{\prime}} \times \mathbb{P}^{r}$, we denote by $p_{X}^{\prime}$ and $p_{X}$ the restrictions to $X$ of $p^{\prime}$ and $p$, respectively. In particular, $p_{S}^{\prime}$ and $p_{S}$ are the maps defined by $\left|R^{\prime}\right|$ and $|R|$, respectively.

We use the standard notation $\mathcal{O}_{\mathbb{P}^{r^{\prime}} \times \mathbb{P} r}(a, b):=p^{\prime *} \mathcal{O}_{\mathbb{P} r^{\prime}}(a) \otimes p^{*} \mathcal{O}_{\mathbb{P}^{r}}(b)$, and for any subvariety $X \subset \mathbb{P}^{r^{\prime}} \times \mathbb{P}^{r}$, we set $\left.\mathcal{O}_{X}(a, b) \simeq \mathcal{O}_{\mathbb{P}^{r^{\prime}} \times \mathbb{P}^{r}}(a, b)\right|_{X}$ and refer to elements in the corresponding linear systems as divisors of bidegree $(a, b)$ on $X$.

Definition 2.6. We say that a curve in $\mathbb{P}^{r^{\prime}} \times \mathbb{P}^{r}$ is vertical if it is contracted by $p^{\prime}$ and horizontal if it is contracted by $p$.

Any line $\ell$ in $\mathbb{P}^{r^{\prime}} \times \mathbb{P}^{r}$ is either vertical or horizontal. If $g \equiv 1 \bmod 4$, then

- $N_{1}, \ldots, N_{4}$ are vertical, as $N_{1} \cdot R^{\prime}=\cdots=N_{4} \cdot R^{\prime}=0$;

- $N_{5}, \ldots, N_{8}$ are horizontal, as $N_{5} \cdot R=\cdots=N_{8} \cdot R=0$.

If instead $g \equiv 3 \bmod 4$, then

- $N_{1}, N_{2}$ are vertical, as $N_{1} \cdot R^{\prime}=N_{2} \cdot R^{\prime}=0$;

- $N_{3}, \ldots, N_{8}$ are horizontal, as $N_{3} \cdot R=\cdots=N_{8} \cdot R=0$.

We will make use of the following:

Lemma 2.7. Let $(S, M, H)$ be a general non-standard Nikulin surface of genus $g$ with $5 \leq g \leq 15$ and let $L, R$ and $R^{\prime}$ be as above. Then the multiplication map

$$
\mu_{R, R^{\prime}}: H^{0}(S, R) \otimes H^{0}\left(S, R^{\prime}\right) \longrightarrow H^{0}(S, L)
$$

is surjective. Furthermore, it is isomorphic to the multiplication map

$$
\mu_{\eta, \eta^{\prime}}: H^{0}(C, \eta) \otimes H^{0}\left(C, \eta^{\prime}\right) \longrightarrow H^{0}\left(C, \eta \otimes \eta^{\prime}\right),
$$

where $C$ is any smooth irreducible curve in $|H|$ and $\eta$ and $\eta^{\prime}$ are the restrictions to $C$ of the line bundles $R$ and $R^{\prime}$, respectively.

Proof. The properties are open in the moduli space, so we may assume that rk $\operatorname{Pic} S=9$. The surjectivity of $\mu_{R, R^{\prime}}$ follows from the generalization by Mumford of a theorem of Castelnuovo, cf. [Mu, Thm. 2, p. 41] (recalling that the assumption on ampleness is unnecessary) once we check that $h^{1}\left(R-R^{\prime}\right)=0$ and $h^{2}\left(R-2 R^{\prime}\right)=0$.

We have $2\left(R-R^{\prime}\right) \sim-N_{1}-\cdots-N_{4}+N_{5}+\cdots+N_{8}\left(\right.$ resp., $\left.-N_{1}-N_{2}+N_{3}+\cdots+N_{8}\right)$ if $g \equiv 1 \bmod 4($ resp., $g \equiv 3 \bmod 4)$. Hence, $h^{0}\left(R-R^{\prime}\right)=h^{0}\left(R^{\prime}-R\right)=0$. As $\left(R-R^{\prime}\right)^{2}=-4$, one has $h^{1}\left(R-R^{\prime}\right)=0$.

We next prove that $h^{2}\left(R-2 R^{\prime}\right)=h^{0}\left(2 R^{\prime}-R\right)=0$. We treat the case $g \equiv 1 \bmod 4$, leaving the other case to the reader, as it is very similar. 
We have $2 R^{\prime}-R \sim R+N_{1}+\cdots+N_{4}-N_{5}-\cdots-N_{8}$. Since $N_{i} \cdot\left(2 R^{\prime}-R\right)=-1$ for $i \in\{1,2,3,4\}$, we have $h^{0}\left(2 R^{\prime}-R\right)=h^{0}\left(R-N_{5}-\cdots-N_{8}\right)$. The assumption on $g$ implies that $\left(R-N_{5}-\cdots-N_{8}\right)^{2} \leq-4$. Hence, if $R-N_{5}-\cdots-N_{8}$ is effective, it has nonvanishing $h^{1}$, which by Ramanujam's vanishing theorem [Ra, Lemma 3] implies that it is not 1connected. Hence, there is an effective nontrivial decomposition $R-N_{5}-\cdots-N_{8} \sim A+B$ such that $A \cdot B \leq 0$. Since Pic $S \simeq \mathbb{Z}[R] \oplus \mathbf{N}$ by [GS, Prop. 2.1 and Cor. 2.1], we may write

$$
A \sim \alpha R+\frac{1}{2} \sum_{i=1}^{8} \alpha_{i} N_{i} \text { and } B \sim \beta R+\frac{1}{2} \sum_{i=1}^{8} \beta_{i} N_{i},
$$

for integers $\alpha, \beta, \alpha_{i}, \beta_{i}$ satisfying

$$
\alpha+\beta=1, \quad \alpha_{i}+\beta_{i}=0 \text { if } i \in\{1,2,3,4\} \text { and } \alpha_{i}+\beta_{i}=-2 \text { if } i \in\{5,6,7,8\} .
$$

Effectivity requires that $\alpha \geq 0$ and $\beta \geq 0$, so that we can without loss of generality assume $\alpha=1$ and $\beta=0$. Therefore, $B \sim \frac{1}{2} \sum_{i=1}^{8} \beta_{i} N_{i}$ and effectivity requires that all $\beta_{i} \geq 0$ and all $\beta_{i}$ are even. Write $\beta_{i}=2 \gamma_{i}$ for integers $\gamma_{i} \geq 0$. Then $\alpha_{i}=-2 \gamma_{i}$ if $i \in\{1,2,3,4\}$ and $\alpha_{i}=-2\left(\gamma_{i}+1\right)$ if $i \in\{5,6,7,8\}$, so that $A \sim R-\sum_{i=1}^{4} \gamma_{i} N_{i}-$ $\sum_{i=5}^{8}\left(\gamma_{i}+1\right) N_{i}$. Therefore,

$$
\begin{aligned}
A \cdot B & =\left(R-\sum_{i=1}^{4} \gamma_{i} N_{i}-\sum_{i=5}^{8}\left(\gamma_{i}+1\right) N_{i}\right) \cdot \sum_{i=1}^{8} \gamma_{i} N_{i} \\
& =\sum_{i=1}^{4} \gamma_{i}\left(2 \gamma_{i}+1\right)+2 \sum_{i=5}^{8} \gamma_{i}\left(\gamma_{i}+1\right) .
\end{aligned}
$$

Since at least one of the $\gamma_{i}$ is strictly positive, we see that we get $A \cdot B \geq 3$, a contradiction.

As concerns the second statement, it is enough to remark that the line bundles $L-H$, $R-H$ and $R^{\prime}-H$ all have vanishing $h^{0}$ and $h^{1}$, which can be proved as in the last part of the proof of Proposition 2.3 .

As a consequence, for $g \geq 7$ the embeddings $C \subset S \subset \mathbb{P}^{r r^{\prime}+r+r^{\prime}}$ in (2) factor through the embedding $S \subset \mathbb{P}^{g-2}$ defined by $|L|$, and hence:

$$
C \subset S \subset\left(\mathbb{P}^{r^{\prime}} \times \mathbb{P}^{r}\right) \cap \mathbb{P}^{g-2} \subset \mathbb{P}^{r r^{\prime}+r+r^{\prime}}
$$

Remark 2.8. It is not a priori obvious that the intersection $\left(\mathbb{P}^{r^{\prime}} \times \mathbb{P}^{r}\right) \cap \mathbb{P}^{g-2}$ is transversal. However, since $\mathcal{F}_{g}^{\mathbf{N}, n s}$ is irreducible, as soon as one shows the existence of a nonstandard Nikulin surface of genus $g$ in some transversal intersection $\left(\mathbb{P}^{r^{\prime}} \times \mathbb{P}^{r}\right) \cap \mathbb{P}^{g-2}$, one gets the transversality statement for a general Nikulin surface in $\mathcal{F}_{g}^{\mathbf{N}, n s}$.

The next two results prove Theorem 1.1 in genera 3 and 5 .

Theorem 2.9. The image of $m_{3}^{\mathbf{N}, n s}$ coincides with the hyperelliptic locus in $\mathcal{M}_{3}$. In particular, a general fiber of $m_{3}^{\mathbf{N}, n s}$ has dimension 9.

Proof. Let $(S, M, H) \in \mathcal{F}_{3}^{\mathbf{N}, n s}$ and $C \in|H|$ be general. The restriction of the line bundle $R \in \operatorname{Pic} S$ to $C$ is a $g_{2}^{1}$; in particular, the canonical map of $C$ is a double cover of a plane conic $C_{K}$ branched along 8 points. Furthermore, the linear system $|H|$ on $S$ defines a double cover $\varphi_{H}: S \rightarrow X \subset \mathbb{P}^{3}$ of a cone $X$ in $\mathbb{P}^{3}$ branched along a plane conic $C_{2}$ that is the image of the unique curve in $\left|R^{\prime}\right|$, and a sextic $C_{6}$ that is the image of an 
irreducible curve in the linear system $\left|H+R^{\prime}\right|$ (cf. [GS, 4.3]). Note that $C_{2} \cdot C_{K}=2$, $C_{6} \cdot C_{K}=6$ and that $C_{2}$ and $C_{6}$ meet at the six points in $X$ that are images of the curves $N_{3}, \ldots, N_{8}$. Furthermore, $\varphi_{H}$ factors through

$$
S \stackrel{c}{\longrightarrow} \bar{S} \stackrel{\pi}{\longrightarrow} \mathbb{F}_{2} \stackrel{\phi}{\longrightarrow} X,
$$

where $\mathbb{F}_{2}$ is the second Hirzebruch surface (with a section $C_{0}$ such that $C_{0}^{2}=-2$ and class fiber denoted by $f$ ), the map $\phi$ is induced by the linear system $\left|C_{0}+2 f\right|$ on $\mathbb{F}_{2}$, the map $\pi$ is a double cover branched along the inverse image of $C_{2}$ and $C_{6}$, while $c$ is the contraction of $N_{3}, \ldots, N_{8}$. Note that $(\pi \circ c)^{-1}\left(C_{0}\right)=N_{1} \cup N_{2}$, and $\phi^{*} C_{2} \in\left|C_{0}+2 f\right|$ while $\phi^{*} C_{6} \in\left|3 C_{0}+6 f\right|$.

It is not difficult to show that the desingularization $S$ of any double cover $\bar{S}$ of $\mathbb{F}_{2}$ branched along the union of a smooth irreducible curve $C_{2} \in\left|C_{0}+2 f\right|$ and a smooth irreducible curve $C_{6} \in\left|3 C_{0}+6 f\right|$ is a Nikulin surface. Indeed, $S$ is a $K 3$ surface by, e.g., [Re, Thm. 2.2]; furthermore, $S$ has eight disjoint rational curves, two of which mapping to the section $C_{0}$ (call them $N_{1}$ and $N_{2}$ ) and six arising as exceptional divisors of the desingularization of $\bar{S}$ (call them $N_{3}, \ldots, N_{8}$ ), which has six double points at the inverse images of $C_{2} \cap C_{6}$. The line bundle $H \in \operatorname{Pic} S$ obtained as pullback of $C_{0}+2 f$ is a genus 3 polarization. We denote by $R \in \operatorname{Pic} S$ the pullback of $f$, and by $R^{\prime} \in \operatorname{Pic} S$ the line bundle with a section vanishing at the strict transform in $S$ of the ramification curve $\pi^{-1}\left(C_{2}\right) \subset \bar{S}$. In particular, we have

$$
H-N_{1}-N_{2} \sim(\pi \circ c)^{*}\left(C_{0}+2 f\right)-(\pi \circ c)^{*} C_{0} \sim 2 R .
$$

Setting $M:=H-R-R^{\prime}$, one easily checks that $N_{1}+\cdots+N_{8} \sim 2 M$ and hence $(S, M, H)$ is a genus 3 Nikulin surface of non-standard type by (6); it depends on $\operatorname{dim}\left|C_{0}+2 f\right|+$ $\operatorname{dim}\left|3 C_{0}+6 f\right|-\operatorname{dim} \operatorname{Aut}\left(\mathbb{F}_{2}\right)=3+15-7=11$ moduli.

We use this in order to prove that a general hyperelliptic curve of genus 3 lies on a Nikulin surface of non-standard type. Let $C$ be a general hyperelliptic curve of genus 3 and let $C_{K} \subset \mathbb{P}^{2}$ be the canonical image of $C$, which is a smooth plane conic. We denote by $x_{1}, \ldots, x_{8}$ the image in $C_{K}$ of the eight Weierstrass points on $C$ and by $X$ the cone in $\mathbb{P}^{3}$ over $C_{K}$. The desingularization of $X$ is then isomorphic to $\mathbb{F}_{2}$. By abuse of notation, we still denote by the same name the inverse images in $\mathbb{F}_{2}$ of the curve $C_{K}$ and the points $x_{1}, \ldots, x_{8}$. It is then enough to remark that both the linear systems $\left|\left(C_{0}+2 f\right) \otimes \mathcal{I}_{x_{1}+x_{2}}\right|$ and $\left|\left(3 C_{0}+6 f\right) \otimes \mathcal{I}_{x_{3}+\cdots+x_{8}}\right|$ are nonempty and contain smooth members.

Theorem 2.10. The map $m_{5}^{\mathbf{N}, n s}$ has generically 6-dimensional fibers and its image coincides with the locus of curves in $\mathcal{M}_{5}$ possessing two autoresidual $g_{4}^{1}$. In particular, this locus is irreducible of dimension 10.

Proof. By [GS, 4.6(b)], the nodal model of a Nikulin surface $S$ of non-standard type and genus 5 is the complete intersection in $\mathbb{P}^{5}$ of three quadrics $Q_{1}, Q_{2}, Q_{3}$ such that $Q_{3}$ is smooth, while $Q_{1}$ and $Q_{2}$ have rank 3 and disjoint singular loci.

Vice versa, we are going to show that the minimal desingularization of any complete intersection $\bar{S}=Q_{1} \cap Q_{2} \cap Q_{3}$ of three quadrics in $\mathbb{P}^{5}$ with the above properties is automatically a Nikulin surface of non-standard type and genus 5 . For $i=1,2$, let $\pi_{i}$ be the plane vertex of $Q_{i}$. The plane $\pi_{1}$ (respectively, $\pi_{2}$ ) intersects $S$ at four nodes $P_{1}, \ldots, P_{4}$ (resp., $\left.P_{5}, \ldots P_{8}\right)$. Let $q: S \rightarrow \bar{S}$ be the minimal desingularization of $\bar{S}$ and let $N_{i}:=q^{-1}\left(P_{i}\right)$ for $1 \leq i \leq 8$. The line bundle $H:=q^{*}\left(\mathcal{O}_{\bar{S}}(1)\right)$ is a genus 5 
polarization on $S$. Up to a change of coordinates, the quadrics $Q_{1}$ and $Q_{2}$ have defining equations $z_{0} z_{1}-z_{2}^{2}=0$ and $z_{3} z_{4}-z_{5}^{2}=0$, respectively; hence, $\pi_{1}: z_{0}=z_{1}=z_{2}=0$ and $\pi_{2}: z_{3}=z_{4}=z_{5}=0$. The hyperplanes $z_{0}=0$ and $z_{1}=0$ generate a pencil of hyperplanes in $\mathbb{P}^{5}$ all passing through the points $P_{1}, \ldots, P_{4}$ and cutting out on $\bar{S}$ a curve with multiplicity two; therefore, there exists a line bundle $R \in \operatorname{Pic} S$ such that $2 R \sim H-N_{1}-N_{2}-N_{3}-N_{4}$. Analogously, one shows the existence of a line bundle $R^{\prime} \in$ Pic $S$ such that $2 R^{\prime} \sim H-N_{5}-N_{6}-N_{7}-N_{8}$. Hence, $S$ is a Nikulin surface of non-standard type.

We are now ready to detect the image of $m_{5}^{\mathbf{N}, n s}$. First of all, it is straightforward that the line bundles $R$ and $R^{\prime}$ on a genus 5 Nikulin surface of non-standard type cut out two autoresidual $g_{4}^{1}$ on a general hyperplane section. The other way around, let us consider a genus 5 curve $C$ possessing two autoresidual $g_{4}^{1}$; these determine two rank-3 quadrics $q_{1}$ and $q_{2}$ in $\mathbb{P}^{4}$ containing the canonical image of $C$, cf. [ACGH, p. 208]. Since any component of the locus in $\mathcal{M}_{5}$ of curves with two autoresidual $g_{4}^{1}$ has dimension at least 10, we can assume $C$ not to be bielliptic; this ensures that the singular lines of $q_{1}$ and $q_{2}$ do not intersect, cf. [ACGH, ch. VI, F]. Fix an embedding $\mathbb{P}^{4} \subset \mathbb{P}^{5}$ and let $Q_{1}$ (respectively, $Q_{2}$ ) be the cone over $q_{1}$ (resp. $q_{2}$ ) with vertex a point $P_{1}$ (resp., $P_{2}$ ) in $\mathbb{P}^{5} \backslash \mathbb{P}^{4}$; then, both $Q_{1}$ and $Q_{2}$ are quadrics of rank 3 and one can choose the points $P_{1}$ and $P_{2}$ so that their singular loci are disjoint. It is easy to check that $h^{0}\left(\mathbb{P}^{5}, \mathcal{I}_{C / \mathbb{P}^{5}}(2)\right)=9$ and a general quadric $Q_{3}$ containing $C$ is smooth since $C$ cannot be trigonal (cf. ACGH, ch. VI, F]); therefore, the surface $\bar{S}=Q_{1} \cap Q_{2} \cap Q_{3}$ is the nodal model of a Nikulin surface of non-standard type. Furthermore, the fiber of $m_{5}^{\mathbf{N}, n s}$ over $[C]$ is parametrized by $\mathbb{P}\left(H^{0}\left(\mathbb{P}^{5}, \mathcal{I}_{C / \mathbb{P}^{5}}(2)\right) /\left\langle Q_{1}, Q_{2}\right\rangle\right)=\mathbb{P}^{6}$.

The rest of the paper will focus on the cases $g=7,9,11$.

Remark 2.11. The following heuristic count shows that the expected dimension of a general fiber of $\chi_{g}^{n s}$ for $g=7,9,11$ is the one obtained in Theorem 1.1.

When $g=7$, a general hyperplane section $C$ carries two theta-characteristics with a space of global sections of dimension 3 and 2, respectively, by Proposition 2.3. The moduli spaces of such curves have codimensions 3 and 1 , respectively, in $\mathcal{M}_{g}$ or $\mathcal{R}_{g}$, by Te, thus one expects the target of $\chi_{7}^{n s}$ to have dimension $18-3-1=14$ and the fibers to have dimension $11+7-14=4$.

When $g=9$, a general hyperplane section $C$ carries two theta-characteristics with a 3-dimensional space of global sections, by Proposition 2.3. The moduli spaces of such curves have codimension 3 in $\mathcal{M}_{g}$ or $\mathcal{R}_{g}$, by [Te], thus one expects the target of $\chi_{9}^{n s}$ to have dimension $24-3-3=18$ and the fibers to have dimension $11+9-18=2$.

When $g=11$, a general hyperplane section $C$ carries two theta-characteristics with 4 and 3 sections, respectively, by Proposition 2.3. The moduli spaces of such curves have codimensions 6 and 3 , respectively, in $\mathcal{M}_{g}$ or $\mathcal{R}_{g}$, by [Te, thus one expects the target of $\chi_{11}^{n s}$ to have dimension $30-6-3=21$ and the fibers to have dimension $11+11-21=1$.

\section{The CASE OF Genus 7}

Let $(S, M, H)$ be a general primitively polarized Nikulin surface of non-standard type of genus 7. Let $L=H-M$ and

$$
R \sim \frac{1}{2}\left(H-N_{1}-N_{2}\right) \text { and } R^{\prime} \sim L-R \sim \frac{1}{2}\left(H-N_{3}-\cdots-N_{8}\right)
$$


be as in 92 . By Proposition 2.3, the line bundle $L$ defines an embedding $S \subset \mathbb{P}^{5}$ and the embeddings in (4) are as follows:

$$
S \subset \mathbb{P}^{1} \times \mathbb{P}^{2} \subset \mathbb{P}^{5}
$$

Here $|R|=\left|\mathcal{O}_{S}(0,1)\right|$ is a net of genus 2 curves of degree $R \cdot L=5$ and $\left|R^{\prime}\right|=\left|\mathcal{O}_{S}(1,0)\right|$ is a pencil of elliptic curves of degree $R^{\prime} \cdot L=3$. By the adjunction formula, $S \in$ $\left|\mathcal{O}_{\mathbb{P}^{1} \times \mathbb{P}^{2}}(2,3)\right|$, cf. [GS, §4.8]. We want to identify the locus in $\left|\mathcal{O}_{\mathbb{P}^{1} \times \mathbb{P}^{2}}(2,3)\right|$ parametrizing Nikulin surfaces of non-standard type. Since $R^{\prime} \cdot N_{1}=R^{\prime} \cdot N_{2}=0$, two elements of $\left|R^{\prime}\right|$ split as $N_{1}+A_{1}$ and $N_{2}+A_{2}$. In particular $A_{1}, A_{2}$ are two disjoint conics in the embedding $S \subset \mathbb{P}^{5}$, mapped into conics in $\mathbb{P}^{2}$ by $p$, as $R \cdot A_{1}=R \cdot A_{2}=2$. Furthermore, one can prove that $A_{1}$ and $A_{2}$ are irreducible by specializing to the case where rk Pic $S=9$ and proceeding as in [GS, proof of Prop. 3.5(2)].

Lemma 3.1. We have $p\left(A_{1}\right)=p\left(A_{2}\right)$.

Proof. Since $N_{j} \cdot R^{\prime}=1$ and $N_{j} \cdot N_{1}=N_{j} \cdot N_{2}=0$ for $j \geq 3$, we have $N_{j} \cdot A_{1}=N_{j} \cdot A_{2}=1$ for $j \geq 3$. It is then enough to note that the six points $z_{j}:=p\left(N_{j}\right), j=3, \ldots, 8$, are distinct and belong to both the conics $p\left(A_{1}\right)$ and $p\left(A_{2}\right)$.

We call $A_{1}$ and $A_{2}$ the vertical conics of $S$.

Using the fact that $R^{\prime} \sim N_{1}+A_{1} \sim N_{2}+A_{2} \sim \frac{1}{2}\left(H-N_{3}-\cdots-N_{8}\right)$, we obtain $2 R^{\prime} \sim\left(N_{1}+A_{1}\right)+\left(N_{2}+A_{2}\right) \sim H-N_{3}-\cdots-N_{8}$, whence

$$
H \sim N_{1}+\cdots+N_{8}+A_{1}+A_{2} .
$$

3.1. Rationality of $\mathcal{F}_{7}^{\mathbf{N}, n s}$. Fix any smooth conic $A \subset \mathbb{P}^{2}$ and two disjoint vertical conics $A_{1}, A_{2} \subset \mathbb{P}^{1} \times \mathbb{P}^{2}$ such that $p\left(A_{1}\right)=p\left(A_{2}\right)=A$. The surface $\mathbb{P}^{1} \times A$ is of bidegree $(0,2)$ in $\mathbb{P}^{1} \times \mathbb{P}^{2}$. Consider the inclusion

$$
\left|\mathcal{I}_{\mathbb{P}^{1} \times A / \mathbb{P}^{1} \times \mathbb{P}^{2}}(2,3)\right| \subset\left|\mathcal{I}_{A_{1} \cup A_{2} / \mathbb{P}^{1} \times \mathbb{P}^{2}}(2,3)\right| .
$$

Proposition 3.2. A general member of $\left|\mathcal{I}_{A_{1} \cup A_{2} / \mathbb{P}^{1} \times \mathbb{P}^{2}}(2,3)\right|$ is smooth and every smooth $S \in\left|\mathcal{I}_{A_{1} \cup A_{2} / \mathbb{P}^{1} \times \mathbb{P}^{2}}(2,3)\right|$ is a non-standard Nikulin surface of genus 7 polarized by $\mathcal{O}_{S}(2,2)\left(-A_{1}-A_{2}\right)$.

Moreover, $\operatorname{dim}\left|\mathcal{I}_{A_{1} \cup A_{2} / \mathbb{P}^{1} \times \mathbb{P}^{2}}(2,3)\right|=15$ and $\operatorname{dim}\left|\mathcal{I}_{\mathbb{P}^{1} \times A / \mathbb{P}^{1} \times \mathbb{P}^{2}}(2,3)\right|=8$.

Proof. The standard exact sequence

$$
0 \longrightarrow \mathcal{I}_{\mathbb{P}^{1} \times A / \mathbb{P}^{1} \times \mathbb{P}^{2}}(2,3) \longrightarrow \mathcal{I}_{A_{1} \cup A_{2} / \mathbb{P}^{1} \times \mathbb{P}^{2}}(2,3) \longrightarrow \mathcal{I}_{A_{1} \cup A_{2} / \mathbb{P}^{1} \times A}(2,3) \longrightarrow 0
$$

along with the isomorphisms $\mathcal{I}_{\mathbb{P}^{1} \times A / \mathbb{P}^{1} \times \mathbb{P}^{2}} \simeq \mathcal{O}_{\mathbb{P}^{1} \times \mathbb{P}^{2}}(0,-2)$ and $\mathcal{I}_{A_{1} \cup A_{2} / \mathbb{P}^{1} \times A}(2,3) \simeq$ $\mathcal{O}_{\mathbb{P}^{1} \times \mathbb{P}^{1}}(-2,0) \otimes \mathcal{O}_{\mathbb{P}^{1} \times \mathbb{P}^{1}}(2,6) \simeq \mathcal{O}_{\mathbb{P}^{1} \times \mathbb{P}^{1}}(0,6)$ proves the dimensional statements, the global generation of $\mathcal{I}_{A_{1} \cup A_{2} / \mathbb{P}^{1} \times \mathbb{P}^{2}}(2,3)$ and the surjectivity of the restriction map of linear systems

$$
\rho:\left|\mathcal{I}_{A_{1} \cup A_{2} / \mathbb{P}^{1} \times \mathbb{P}^{2}}(2,3)\right| \longrightarrow\left(A_{1} \cup A_{2}\right)+\left|\mathcal{O}_{\mathbb{P}^{1} \times \mathbb{P}^{1}}(0,6)\right| .
$$

Hence, a general $S \in\left|\mathcal{I}_{A_{1} \cup A_{2} / \mathbb{P}^{1} \times \mathbb{P}^{2}}(2,3)\right|$ is smooth and

$$
S \cdot\left(\mathbb{P}^{1} \times A\right)=A_{1}+A_{2}+N_{3}+\cdots+N_{8} \in\left|\mathcal{O}_{S}(0,2)\right|,
$$

with $N_{3}, \ldots, N_{8}$ disjoint horizontal lines. At the same time, $\left|\mathcal{O}_{S}(1,0)\right|$ is a pencil of elliptic curves of degree 3 on $S$ such that $\mathcal{O}_{S}(1,0) \cdot A_{i}=0$ for $i=1,2$, and hence contains two elements of the form $N_{i}+A_{i}$ with $N_{i}$ a line for $i=1,2$. Furthermore, $N_{1}$ and $N_{2}$ are mutually disjoint, as well as disjoint from the other $N_{j}$ for $j=3, \ldots, 8$. 
Note that the divisor $N_{1}+\cdots+N_{8} \in\left|\mathcal{O}_{S}(2,2)\left(-2 A_{1}-2 A_{2}\right)\right|$ and thus is 2-divisible in Pic $S$. It is now straightforward that $S$ satisfies the desired properties; in particular, (7) implies that $S$ is of non-standard type.

Two smooth elements in $\left|\mathcal{I}_{A_{1} \cup A_{2} / \mathbb{P}^{1} \times \mathbb{P}^{2}}(2,3)\right|$ are isomorphic if and only if they are in the same orbit under the action of the stabilizer $G$ of $A_{1} \cup A_{2}$ in $\operatorname{Aut}\left(\mathbb{P}^{1} \times A\right)$. The group $G$ is 4-dimensional, since it is the product of the stabilizer of two points in $\mathbb{P}^{1}$ and of the group Aut $A$. Hence the quotient $\left|\mathcal{I}_{A_{1} \cup A_{2} / \mathbb{P}^{1} \times \mathbb{P}^{2}}(2,3)\right| / G$ is 11 -dimensional and we have a birational map

$$
\left|\mathcal{I}_{A_{1} \cup A_{2} / \mathbb{P}^{1} \times \mathbb{P}^{2}}(2,3)\right| / G-->\mathcal{F}_{7}^{\mathbf{N}, n s} .
$$

Theorem 3.3. The moduli space $\mathcal{F}_{7}^{\mathbf{N}, n s}$ is rational.

Proof. The blow-up of $\mathbb{P}^{15}:=\left|\mathcal{I}_{A_{1} \cup A_{2} / \mathbb{P}^{1} \times \mathbb{P}^{2}}(2,3)\right|$ along $\mathbb{P}^{8}:=\left|\mathcal{I}_{\mathbb{P}^{1} \times A / \mathbb{P}^{1} \times \mathbb{P}^{2}}(2,3)\right|$ is a $\mathbb{P}^{9}$-bundle $\pi: \mathbb{P} \rightarrow \mathbb{P}^{6}$. Let $o \in \mathbb{P}^{6}$, then $\pi^{-1}(o)$ is a 9 -dimensional linear system generated by $\mathbb{P}^{8}$ and by an element $S \in \mathbb{P}^{15}$ not containing $\mathbb{P}^{1} \times A$. It is useful to remark that then the base locus of $\pi^{-1}(o)$ is $S \cdot\left(\mathbb{P}^{1} \times A\right)=A_{1}+A_{2}+N_{o 1}+\cdots+N_{o 6}$, where the last six summands are the 'horizontal' lines in the surface $S$. Let $p_{A}^{\prime}: \mathbb{P}^{1} \times A \rightarrow A$ be the projection map. Since $N_{o 1}+\cdots+N_{o 6} \in\left|\mathcal{O}_{A}(3)\right|$, this yields an immediate identification

$$
\mathbb{P}^{6}:=\left|\mathcal{O}_{A}(3)\right|=\left|\mathcal{O}_{\mathbb{P}^{1}}(6)\right|,
$$

under the linear isomorphism sending $o$ to $n:=\left(p_{A}^{\prime}\right)_{*}\left(N_{o 1}+\cdots+N_{o 6}\right)$. Now it is clear that $G$ acts linearly on $\mathbb{P}$ and on $\mathbb{P}^{6}$. Furthermore, by Castelnuovo's criterion, $\mathbb{P}^{6} / G$ is a unirational surface, hence it is rational. To complete the proof it suffices to show that $\mathbb{P} / G$ is a $\mathbb{P}^{9}$-bundle over a nonempty open set of $\mathbb{P} / G$. Let $U \subset \mathbb{P}^{6}$ be the open set of the degree six divisors $n \in\left|\mathcal{O}_{A}(3)\right|$ such that the stabilizer of $n$ in Aut $A$ is trivial; this is nonempty since there are no non-trivial automorphisms of $\mathbb{P}^{1}$ mapping a set of 6 general points to itself. This immediately implies that, whenever $o \in U$, the stabilizer of $\pi^{-1}(o)$ in $G$ is trivial: otherwise $n$ would be invariant under the action of some non trivial $\gamma \in G$. Let $\mathbb{P}_{U}$ be the restriction of $\mathbb{P}$ to $U$. Since the stabilizer of $\pi^{-1}(o)$ is trivial along $U$, it follows from Kempf's descent lemma, cfr. [DN], that $\mathbb{P}_{U}$ descends to a $\mathbb{P}^{9}$-bundle $\mathbb{P}_{U} / G$ over $U / G$. This implies the statement.

3.2. The fibre of the Prym-Nikulin map $\chi_{7}^{n s}$. We start with a general point $(S, M, H)$ in $\mathcal{F}_{7}^{\mathbf{N}, n s}$ and a general smooth $C \in|H|$. We still denote by $A_{1}$ and $A_{2}$ the two vertical conics of $S$.

Lemma 3.4. We have

(i) $h^{0}\left(\mathcal{I}_{C / \mathbb{P}^{1} \times \mathbb{P}^{2}}(2,2)\right)=h^{1}\left(\mathcal{I}_{C / \mathbb{P}^{1} \times \mathbb{P}^{2}}(2,2)\right)=1$,

(ii) $C$ is not quadratically normal in $\mathbb{P}^{5}$,

(iii) $h^{0}\left(\mathcal{I}_{C / \mathbb{P}^{1} \times \mathbb{P}^{2}}(2,3)\right)=6$,

(iv) $h^{0}\left(\mathcal{I}_{C \cup A_{1} \cup A_{2} / \mathbb{P}^{1} \times \mathbb{P}^{2}}(2,3)\right)=4$.

Proof. Item (i) follows from the exact sequence

$$
0 \longrightarrow \mathcal{I}_{S / \mathbb{P}^{1} \times \mathbb{P}^{2}} \simeq \mathcal{O}_{\mathbb{P}^{1} \times \mathbb{P}^{2}}(-2,-3) \longrightarrow \mathcal{I}_{C / \mathbb{P}^{1} \times \mathbb{P}^{2}} \longrightarrow \mathcal{I}_{C / S} \simeq \mathcal{O}_{S}(-H) \longrightarrow 0
$$

tensored by $\mathcal{O}_{\mathbb{P}^{1} \times \mathbb{P}^{2}}(2,2)$ and the isomorphisms

$$
\mathcal{O}_{S}(2 L-H) \simeq \mathcal{O}_{S}(H-2 M) \simeq \mathcal{O}_{S}\left(A_{1}+A_{2}\right),
$$


cf. (7). Item (ii) is an immediate consequence of (i).

Item (iii) follows from the above sequence tensored by $\mathcal{O}_{\mathbb{P}^{1} \times \mathbb{P}^{2}}(2,3)$ and the equality $h^{0}\left(S, R+A_{1}+A_{2}\right)=5$. Item (iv) follows similarly.

Remark 3.5. Lemma 3.4(ii) is of particular interest. Indeed, it implies that the image of the moduli map $\chi_{7}^{n s}: \mathcal{P}_{7}^{\mathbf{N}, n s} \rightarrow \mathcal{R}_{7}$ lies in the ramification locus of the Prym map $\mathcal{R}_{7} \rightarrow \mathcal{A}_{6}$, cf. [Be].

Theorem 1.1 in genus 7 follows by detecting the locus $\mathcal{D}_{C}$ in $\left|\mathcal{I}_{C / \mathbb{P}^{1} \times \mathbb{P}^{2}}(2,3)\right|$ that parametrizes Nikulin surfaces of non-standard type.

Theorem 3.6. The fibre of $\chi_{7}^{n s}: \mathcal{F}_{7}^{\mathbf{N}, n s} \rightarrow \mathcal{R}_{7}$ over $C$ is 4-dimensional.

Proof. We consider the 5-dimensional linear system $\left|\mathcal{I}_{C / \mathbb{P}^{1} \times \mathbb{P}^{2}}(2,3)\right|$, cf. Lemma 3.4(iii), along with its linear subsystem $\left|\mathcal{I}_{C \cup A_{1} \cup A_{2} / \mathbb{P}^{1} \times \mathbb{P}^{2}}(2,3)\right| \subset\left|\mathcal{I}_{C / \mathbb{P}^{1} \times \mathbb{P}^{2}}(2,3)\right|$, which has dimension 3 and parametrizes Nikulin surfaces of non-standard type by Lemma 3.4(iv) and Proposition 3.2

We are going to show the existence of a one-dimensional family of such linear subsystems, the union of which is a hypersurface $\mathcal{D}_{C}$ in $\left|\mathcal{I}_{C / \mathbb{P}^{1} \times \mathbb{P}^{2}}(2,3)\right|$ parametrizing Nikulin surfaces of non-standard type.

Lemma 3.4(i) yields that $C \subset Y \subset \mathbb{P}^{1} \times \mathbb{P}^{2}$, where $Y$ is integral of bidegree $(2,2)$. The linear system $\left|\mathcal{O}_{Y}(1,0)\right|$ is a ruling of conics on $Y$, and $A_{1}, A_{2}$ are in this ruling, since $C \subset Y$ and $A_{j} \cdot C=6$. For each $x \in \mathbb{P}^{1}$ we denote by $A_{x}$ the conic over the point $x$. Consider the map

$$
p_{*}:\left|\mathcal{O}_{Y}(1,0)\right| \longrightarrow\left|\mathcal{O}_{\mathbb{P}^{2}}(2)\right|,
$$

sending $A_{x}$ to $p_{*} A_{x}$. Since $p_{Y}: Y \rightarrow \mathbb{P}^{2}$ has degree two, the map $p_{*}$ has degree one or two. As $p_{*} A_{1}=p_{*} A_{2}=A$, it has degree two. Hence there exists an involution $\iota: \mathbb{P}^{1} \rightarrow \mathbb{P}^{1}$ such that $p_{*} A_{x}=p_{*} A_{\iota(x)}$. Thus we have a fibration

$$
\mathcal{D}_{C} \longrightarrow \mathbb{P}^{1},
$$

sending a surface $S$ to the pair of conjugated points defined by its vertical conics; in other words, the base $\mathbb{P}^{1}$ is the quotient of $\left|\mathcal{O}_{Y}(1,0)\right|$ by the involution $\iota$ and the fiber over a point $\langle x, \iota(x)\rangle \in \mathbb{P}^{1}$ is the 3 -dimensional linear subsystem $\left|\mathcal{I}_{A_{x} \cup A_{\iota(x)} \cup C / \mathbb{P}^{1} \times \mathbb{P}^{2}}(2,3)\right| \subset$ $\left|\mathcal{I}_{C / \mathbb{P}^{1} \times \mathbb{P}^{2}}(2,3)\right|$. Hence $\mathcal{D}_{C}$ is 4-dimensional.

It remains to show that the moduli map $m_{C}: \mathcal{D}_{C} \rightarrow-\rightarrow \mathcal{F}_{7}^{\mathbf{N}, n s}$ is generically finite. This easily follows since there are finitely many automorphism of $\mathbb{P}^{1} \times \mathbb{P}^{2}$ fixing $C$; indeed, any of them different from the identity would induce a non-trivial automorphism of $C$ itself.

\section{The CASE OF Genus 9}

Let $(S, M, H)$ be a general primitively polarized Nikulin surface of non-standard type of genus 9. Let $L=H-M$ and

$$
R \sim \frac{1}{2}\left(H-N_{1}-N_{2}-N_{3}-N_{4}\right) \text { and } R^{\prime} \sim L-R \sim \frac{1}{2}\left(H-N_{5}-N_{6}-N_{7}-N_{8}\right)
$$

be as in 92 , We have $R^{2}=R^{\prime 2}=2$ and $R \cdot R^{\prime}=4$. By Proposition 2.3 , the line bundle $L$ defines an embedding $S \subset \mathbb{P}^{7}$ and $|R|$ and $\left|R^{\prime}\right|$ are base point free linear systems whose 
general member is a smooth, irreducible curve of genus 2. As in (44), the embeddings $C \subset S \subset \mathbb{P}^{7}$ thus factor as

$$
S \subset\left(\mathbb{P}^{2} \times \mathbb{P}^{2}\right) \cap \mathbb{P}^{7} \subset \mathbb{P}^{8}
$$

We may assume that the intersection

$$
T:=\left(\mathbb{P}^{2} \times \mathbb{P}^{2}\right) \cap \mathbb{P}^{7}
$$

is transversal (cf. Remark 2.8 and Proposition 4.3 below) and hence a sextic Del Pezzo threefold. Since $\omega_{T} \simeq \mathcal{O}_{T}(-2,-2)$, we have, by adjunction, cf. [GS, §4.9]:

Lemma 4.1. The surface $S$ is the complete intersection in $\mathbb{P}^{2} \times \mathbb{P}^{2}$ of a hyperplane section and of a quadratic section defined by a quadric $Q$ :

$$
S=Q \cap \mathbb{P}^{7} \cap\left(\mathbb{P}^{2} \times \mathbb{P}^{2}\right)=Q \cap T \subset \mathbb{P}^{8} .
$$

The first and second projections $p_{S}^{\prime}: S \rightarrow \mathbb{P}^{2}$ and $p_{S}: S \rightarrow \mathbb{P}^{2}$ are double coverings of $\mathbb{P}^{2}$, contracting the set of lines $\left\{N_{1}, \ldots, N_{4}\right\}$ and $\left\{N_{5}, \ldots, N_{8}\right\}$, respectively.

The line bundle

$$
E:=H-N_{1}-\cdots-N_{8}
$$

plays a crucial role.

Lemma 4.2. The linear system $|E|$ is an elliptic pencil on $S$. Furthermore, for any $F \in|E|$, we have:

(i) The maps $p_{F}^{\prime}: F \rightarrow \mathbb{P}^{2}$ and $p_{F}: F \rightarrow \mathbb{P}^{2}$ are double coverings onto smooth conics $A^{\prime}$ and $A$, respectively;

(ii) $F=\left(A^{\prime} \times A\right) \cap \mathbb{P}^{7} \subset\left(\mathbb{P}^{2} \times \mathbb{P}^{2}\right) \cap \mathbb{P}^{7}=T$.

(iii) The two surfaces $Y^{\prime}:=\left(A^{\prime} \times \mathbb{P}^{2}\right) \cap \mathbb{P}^{7}$ and $Y:=\left(\mathbb{P}^{2} \times A\right) \cap \mathbb{P}^{7}$ are minimal sextic scrolls (isomorphic to $\mathbb{P}^{1} \times \mathbb{P}^{1}$ ) embedded in $T$ such that $F=Y^{\prime} \cap Y$ and $F$ is anticanonical in $Y^{\prime}$ and $Y$. Moreover, $N_{1} \cup \cdots \cup N_{4} \subset Y^{\prime}$ and $N_{5} \cup \cdots \cup N_{8} \subset Y$.

Proof. Using the fact that $\operatorname{rk} \operatorname{Pic} S=9$, it is easy to check that $E$ is nef and primitive, whence an elliptic pencil. Let $F \in|E|$. As $p_{S}$ has degree two, $p_{F}$ is either birational or of degree two onto its image. In the former case the image would be a quartic curve, as $R \cdot E=4$; however, $p$ contracts $N_{i}, i=1,2,3,4$, and $N_{i} \cdot E=2$, so the quartic would have four singular points, a contradiction. The same works for $p_{F}^{\prime}$. Hence, (i) is proved.

Letting $A=p(F)$ and $A^{\prime}=p^{\prime}(F)$, we have

$$
F \subset\left(A \times A^{\prime}\right) \subset\left(\mathbb{P}^{2} \times \mathbb{P}^{2}\right) \cap \mathbb{P}^{7}=T
$$

Moreover, $A \times A^{\prime}$ is the 2 -Veronese embedding of $\mathbb{P}^{1} \times \mathbb{P}^{1}$ defined by $\left|\mathcal{O}_{\mathbb{P}^{1} \times \mathbb{P}^{1}}(2,2)\right|$. Hence $F$ is a hyperplane section of it, proving (ii). Property (iii) easily follows since the projection $p_{Y}^{\prime}: Y^{\prime} \rightarrow A^{\prime}$ realizes $Y^{\prime}$ as the $\mathbb{P}^{1}$-bundle $\mathbb{P}\left(\mathcal{O}_{\mathbb{P}^{1}}(3) \oplus \mathcal{O}_{\mathbb{P}^{1}}(3)\right)$ over $A^{\prime} \simeq \mathbb{P}^{1}$, and similarly for $Y$.

4.1. A rational parametrization of a double cover of $\mathcal{F}_{9}^{\mathbf{N}, n s}$. Let us fix a Del Pezzo threefold $T:=\left(\mathbb{P}^{2} \times \mathbb{P}^{2}\right) \cap \mathbb{P}^{7} \subset \mathbb{P}^{8}$. Since $T$ is smooth, the restriction map $\operatorname{Pic}\left(\mathbb{P}^{2} \times \mathbb{P}^{2}\right) \rightarrow \operatorname{Pic} T$ is an isomorphism by the Lefschetz Theorem, whence $T$ contains no plane. In particular, both projections $p_{T}^{\prime}: T \rightarrow \mathbb{P}^{2}$ and $p_{T}: T \rightarrow \mathbb{P}^{2}$ realize $T$ as a $\mathbb{P}^{1}$-bundle over $\mathbb{P}^{2}$. We fix four vertical lines $N_{1}, \ldots, N_{4}$ and four horizontal lines $N_{5}, \ldots, N_{8}$ in $T$ such that the points $p^{\prime}\left(N_{1}\right), \ldots, p^{\prime}\left(N_{4}\right)$ are in general position, and the same for $p\left(N_{5}\right), \ldots p\left(N_{8}\right)$. 
Proposition 4.3. A general member of $\left|\mathcal{I}_{N_{1} \cup \ldots \cup N_{8} / T}(2,2)\right|$ is smooth and every smooth $S \in\left|\mathcal{I}_{N_{1} \cup \ldots \cup N_{8} / T}(2,2)\right|$ is a non-standard Nikulin surface of genus 9 polarized by $\mathcal{O}_{S}(2,0)\left(N_{5}+\cdots+N_{8}\right)$.

Moreover, $\operatorname{dim}\left|\mathcal{I}_{N_{1} \cup \cdots \cup N_{8} / T}(2,2)\right|=3$.

Proof. Set

$$
b^{\prime}:=\left\{p^{\prime}\left(N_{1}\right), \ldots, p^{\prime}\left(N_{4}\right)\right\} \text { and } b:=\left\{p\left(N_{5}\right), \ldots p\left(N_{8}\right)\right\} .
$$

and let $A^{\prime}$ (respectively, $A$ ) be any smooth conic passing through $b^{\prime}$ (resp., $b$ ). Define the following surfaces contained in $T$ :

$$
Y^{\prime}:=\left(A^{\prime} \times \mathbb{P}^{2}\right) \cap \mathbb{P}^{7} \in\left|\mathcal{O}_{T}(2,0)\right| \text { and } Y:=\left(\mathbb{P}^{2} \times A\right) \cap \mathbb{P}^{7} \in\left|\mathcal{O}_{T}(0,2)\right|,
$$

which are minimal sextic scrolls isomorphic to $\mathbb{P}^{1} \times \mathbb{P}^{1}$. One easily verifies that $F:=$ $Y^{\prime} \cap Y$ is anticanonical in both $Y^{\prime}$ and $Y$ and that $N_{1} \cup \cdots \cup N_{4} \subset Y^{\prime}$ and $N_{5} \cup \cdots \cup N_{8} \subset Y$. More precisely,

$$
\begin{aligned}
N_{1}+\cdots+N_{4} & \in\left|\mathcal{O}_{Y^{\prime}}(2,0)\right| \simeq\left|\mathcal{O}_{\mathbb{P}^{1} \times \mathbb{P}^{1}}(4,0)\right| \\
N_{5}+\cdots+N_{8} & \in\left|\mathcal{O}_{Y}(0,2)\right| \simeq\left|\mathcal{O}_{\mathbb{P}^{1} \times \mathbb{P}^{1}}(0,4)\right| .
\end{aligned}
$$

We have $\mathcal{I}_{F / Y^{\prime} \cup Y} \simeq \mathcal{I}_{F / Y^{\prime}} \oplus \mathcal{I}_{F / Y}$. Tensoring by $\mathcal{O}_{Y \cup Y^{\prime}}(2,2)$ and using the fact that $F \in\left|\mathcal{O}_{Y^{\prime}}(0,2)\right|$ and $F \in\left|\mathcal{O}_{Y}(2,0)\right|$ by (9), we get

$$
\mathcal{I}_{F / Y^{\prime} \cup Y}(2,2) \simeq \mathcal{O}_{Y^{\prime}}(2,0) \oplus \mathcal{O}_{Y}(0,2) \simeq \mathcal{O}_{\mathbb{P}^{1} \times \mathbb{P}^{1}}(4,0) \oplus \mathcal{O}_{\mathbb{P}^{1} \times \mathbb{P}^{1}}(0,4)
$$

We also have a short exact sequence

$$
0 \longrightarrow \mathcal{I}_{Y^{\prime} \cup Y / T}(2,2) \simeq \mathcal{O}_{T} \longrightarrow \mathcal{I}_{F / T}(2,2) \longrightarrow \mathcal{I}_{F / Y^{\prime} \cup Y}(2,2) \longrightarrow 0,
$$

where the isomorphism follows as $Y^{\prime} \cup Y \in\left|\mathcal{O}_{T}(2,2)\right|$ by (9). From (10) and (11) we get that $\mathcal{I}_{F / T}(2,2)$ is globally generated and the restriction map of linear systems

$$
\left|\mathcal{I}_{F / T}(2,2)\right| \longrightarrow\left(F+\left|\mathcal{O}_{Y^{\prime}}(2,0)\right|\right) \times\left(F+\left|\mathcal{O}_{Y}(0,2)\right|\right)
$$

is surjective. Hence, there is a smooth $S \in\left|\mathcal{I}_{F / T}(2,2)\right|$ containing $N_{1} \cup \cdots \cup N_{8}$, and

$$
\begin{aligned}
S \cdot Y^{\prime} & =N_{1}+\cdots+N_{4}+F \in\left|\mathcal{O}_{S}(2,0)\right| \\
S \cdot Y & =N_{5}+\cdots+N_{8}+F \in\left|\mathcal{O}_{S}(0,2)\right| .
\end{aligned}
$$

In particular, the divisor

$$
N_{1}+\cdots+N_{8} \in\left|\mathcal{O}_{S}(2,2)(-2 F)\right|
$$

is 2-divisible in Pic $S$. It is then easy to see that $S$ is a non-standard Nikulin surface of genus 9 polarized by $\mathcal{O}_{S}(2,0)\left(N_{5}+\cdots+N_{8}\right)$.

Finally, the sequence

$$
0 \longrightarrow \mathcal{I}_{S / T}(2,2) \simeq \mathcal{O}_{T} \longrightarrow \mathcal{I}_{N_{1} \cup \cdots \cup N_{8} / T}(2,2) \longrightarrow \mathcal{I}_{N_{1} \cup \ldots \cup N_{8} / S}(2,2) \simeq \mathcal{O}_{S}(2 F) \longrightarrow 0,
$$

yields $h^{0}\left(\mathcal{I}_{N_{1} \cup \ldots \cup N_{8} / T}(2,2)\right)=4$.

We obtain a nice parametrization of the moduli space $\mathcal{F}_{9}^{\mathbf{N}, n s}$. We fix four vertical lines $N_{1}, \ldots, N_{4}$ in $T$, and observe that in the space of the Segre embedding one has

$$
\left\langle N_{1} \cup \cdots \cup N_{4}\right\rangle=\mathbb{P}^{7}
$$

since $N_{1}, \ldots, N_{4}$ are contained in a minimal sextic scroll $Y^{\prime} \simeq \mathbb{P}^{1} \times \mathbb{P}^{1} \subset \mathbb{P}^{7}$ defined as in the previous proof. It is clear that, up to the action of Aut $T$, we can choose this set of four lines up to the ordering of its elements. Since these four lines are spanning 
$\langle T\rangle=\mathbb{P}^{7}$ and the automorphisms of $T$ are the automorphisms of $\mathbb{P}^{2} \times \mathbb{P}^{2}$ fixing this $\mathbb{P}^{7}$, the stabilizer of $N_{1} \cup N_{2} \cup N_{3} \cup N_{4}$ in Aut $T$ coincides with the stabilizer in $\operatorname{Aut}\left(\mathbb{P}^{2} \times \mathbb{P}^{2}\right)$ of the same set. Recall that

$$
\operatorname{Aut}\left(\mathbb{P}^{2} \times \mathbb{P}^{2}\right) \simeq \operatorname{PGL}(3) \times \operatorname{PGL}(3) \times \mathbb{Z} / 2 \mathbb{Z},
$$

where the $\mathbb{Z} / 2 \mathbb{Z}$-factor is due to the involution interchanging the two factors of $\mathbb{P}^{2} \times \mathbb{P}^{2}$. For $i=1, \ldots, 4$ we have $N_{i}=\left\{o_{i}\right\} \times \ell_{i}$, where $o_{i}=p^{\prime}\left(N_{i}\right)$ is a point and $\ell_{i}=p\left(N_{i}\right)$ is a line. The stabilizer of $N_{1} \cup \cdots \cup N_{4}$ acts on the set of pairs $\left\{\left(o_{1}, \ell_{1}\right), \ldots,\left(o_{4}, \ell_{4}\right)\right\}$. Hence the stabilizer is the diagonal embedding $S_{4} \subset S_{4} \times S_{4}$. The action is the diagonal action: $\alpha\left(o_{i}, \ell_{i}\right)=\left(\alpha\left(o_{i}\right), \alpha\left(\ell_{i}\right)\right)$. We define $N_{1 \ldots 4}:=\left\{N_{1}, \ldots, N_{4}\right\}$ and choose a general set $N_{5 \ldots 8}:=\left\{N_{5}, \ldots, N_{8}\right\}$ of four horizontal lines, or equivalently, four points in $p(T)=\mathbb{P}^{2}$. Then the moduli space of pairs $\left(N_{1 \ldots 4}, N_{5 \ldots 8}\right)$ is precisely the quotient

$$
\left(\mathbb{P}^{2}\right)^{4} / S_{4}
$$

where $S_{4} \subset$ Aut $T$ is the previous group of automorphisms. Hence it acts as above: $\alpha(o, \ell)=(\alpha(o), \alpha(\ell))$ and $\alpha(\ell, o)=(\alpha(\ell), \alpha(o))$. Thus we have:

Theorem 4.4. The quotient $\left(\mathbb{P}^{2}\right)^{4} / S_{4}$ is the 4 -symmetric product of $\mathbb{P}^{2}$ and hence is rational.

For a general pair $\left(N_{1234}, N_{5678}\right)$, with $N_{1 \ldots 4}$ fixed, the linear system $\left|\mathcal{I}_{N_{1} \cup \ldots \cup N_{8} / T}(2,2)\right|$ defines a $\mathbb{P}^{3}$-bundle over $\left(\mathbb{P}^{2}\right)^{4}$. This bundle descends to $\left(\mathbb{P}^{2}\right)^{4} / S_{4}$, thus implying the following:

Theorem 4.5. The moduli space of fourtuples $\left(S, M, H, N_{1234}\right)$ is rational and a double cover of $\mathcal{F}_{9}^{\mathbf{N}, n s}$.

4.2. The fibre of the Prym-Nikulin map $\chi_{9}^{n s}$. Let both $(S, M, H) \in \mathcal{F}_{9}^{\mathbf{N}, n s}$ and $C \in|H|$ be general. Let $E$ be as in (8) and recall Lemma 4.2. The genus 9 case of Theorem 1.1 is a consequence of the next two results.

Lemma 4.6. We have

$$
\operatorname{dim}\left|\mathcal{I}_{C / T}(2,2)\right|=2 .
$$

In particular, $C$ is quadratically normal.

Proof. Fix any $F \in|E|$. Since $2 L \sim C+F$ and $T$ is projectively normal, the curve $C \cup F$ is the complete intersection in $T$ of two quadratic sections. Therefore, we have

$$
h^{0}\left(\mathcal{I}_{C \cup F / T}(2,2)\right)=2 \text { and } h^{1}\left(\mathcal{I}_{C \cup F / T}(2,2)\right)=h^{2}\left(\mathcal{I}_{C \cup F / T}(2,2)\right)=0 .
$$

We consider the standard exact sequence

$$
0 \longrightarrow \mathcal{I}_{C \cup F / T}(2,2) \longrightarrow \mathcal{I}_{C / T}(2,2) \oplus \mathcal{I}_{F / T}(2,2) \longrightarrow \mathcal{I}_{C \cap F / T}(2,2) \longrightarrow 0 .
$$

Taking cohomology in (10) and (11) yields

$$
h^{0}\left(\mathcal{I}_{F / T}(2,2)\right)=11 \text { and } h^{1}\left(\mathcal{I}_{F / T}(2,2)\right)=h^{2}\left(\mathcal{I}_{F / T}(2,2)\right)=0
$$

This, together with the sequence

$$
0 \longrightarrow \mathcal{I}_{F / T}(2,2) \longrightarrow \mathcal{I}_{F \cap C / T}(2,2) \longrightarrow \mathcal{I}_{F \cap C / F}(2,2) \simeq \mathcal{O}_{F}(2 L-C) \longrightarrow 0,
$$

and the fact that $2 L-C \sim F$ and $\mathcal{O}_{F}(F) \simeq \mathcal{O}_{F}$, yields

$$
h^{0}\left(\mathcal{I}_{F \cap C / T}(2,2)\right)=h^{0}\left(\mathcal{I}_{F / T}(2,2)\right)+h^{0}\left(\mathcal{O}_{F}\right)=12 .
$$


Thus, the cohomology of (15) together with (14), (16) and (17) yields $h^{0}\left(\mathcal{I}_{C / T}(2,2)\right)=3$.

The fact that $C$ is quadratically normal is easily checked.

Proposition 4.7. A general $S^{\prime} \in\left|\mathcal{I}_{C / T}(2,2)\right|$ defines a point of $\mathcal{F}_{9}^{\mathbf{N}, n s}$, and the moduli map $\left|\mathcal{I}_{C / T}(2,2)\right| \rightarrow \mathcal{F}_{9}^{\mathbf{N}, n s}$ is generically injective.

Proof. As $S \cdot S^{\prime} \sim 2 L$ on $S$, we have

$$
S^{\prime} \cdot S=F+C \in\left|\mathcal{O}_{S^{\prime}}(2,2)\right|
$$

for some $F \in|E|$. Let $Y^{\prime}$ and $Y$ be as in Lemma 4.2(iii).

Using the fact that $F$ is anticanonical on $Y^{\prime}$, it is not difficult to show that

$$
S^{\prime} \cdot Y^{\prime}=N_{1}^{\prime}+\cdots+N_{4}^{\prime}+F \in\left|\mathcal{O}_{Y^{\prime}}(2,2)\right| \simeq\left|\mathcal{O}_{\mathbb{P}^{1} \times \mathbb{P}^{1}}(6,2)\right|,
$$

with $N_{1}^{\prime}, \ldots, N_{4}^{\prime}$ four disjoint lines in $\left|\mathcal{O}_{\mathbb{P}^{1} \times \mathbb{P}^{1}}(1,0)\right|$. Similarly, one shows that

$$
S^{\prime} \cdot Y=N_{5}^{\prime}+\cdots+N_{8}^{\prime}+F \in\left|\mathcal{O}_{Y}(2,2)\right| \simeq\left|\mathcal{O}_{\mathbb{P}^{1} \times \mathbb{P}^{1}}(2,6)\right|,
$$

with $N_{5}^{\prime}, \ldots, N_{8}^{\prime}$ four disjoint lines in $\left|\mathcal{O}_{\mathbb{P}^{1} \times \mathbb{P}^{1}}(0,1)\right|$. Hence $S^{\prime}$ is a non-standard Nikulin surface of genus 9 by Proposition 4.3 .

We now show that the moduli map $m_{C}:\left|\mathcal{I}_{C / T}(2,2)\right| \rightarrow \mathcal{F}_{9}^{\mathbf{N}, n s}$ is generically injective. Assume that $m_{C}\left(S^{\prime}\right)=m_{C}\left(S^{\prime \prime}\right)$, for distinct $S^{\prime}, S^{\prime \prime} \in\left|\mathcal{I}_{C / T}(2,2)\right|$. Then there exists $\alpha \in \operatorname{Aut}(T)$ such that $\alpha\left(S^{\prime}\right)=S^{\prime \prime}$. In particular, such an $\alpha$ would fix $C$ and thus induce a non-trivial automorphism of $C$. This is a contradiction because the image of $m_{9}^{\mathbf{N}, n s}$ has dimension at least $20-2=18$, while the maximal dimension of a component of the locus in $\mathcal{M}_{9}$ of curves with a non-trivial automorphism is $2 g-1=17$, cf. [Co].

\section{The CASE OF Genus 11}

Let $(S, M, H)$ be a general primitively polarized Nikulin surface of non-standard type of genus 11. Let $L=H-M$, then we have as in $\oiint 2$

$$
R \sim \frac{1}{2}\left(H-N_{1}-N_{2}\right) \text { and } R^{\prime} \sim L-R \sim \frac{1}{2}\left(H-N_{3}-\cdots-N_{8}\right) .
$$

By Proposition 2.3 , the line bundle $L$ defines an embedding $S \subset \mathbb{P}^{9}$. Moreover $|R|$ and $\left|R^{\prime}\right|$ are base point free linear systems, respectively of dimensions 3 and 2 , such that $R^{2}=4, R^{\prime 2}=2$ and $R \cdot R^{\prime}=5$. The embedding $S \subset \mathbb{P}^{9}$ factors as follows

$$
S \subset\left(\mathbb{P}^{2} \times \mathbb{P}^{3}\right) \cap \mathbb{P}^{9} \subset \mathbb{P}^{11},
$$

where the inclusion $\mathbb{P}^{2} \times \mathbb{P}^{3} \subset \mathbb{P}^{11}$ is the Segre embedding and $\mathbb{P}^{9}$ is linearly embedded. We may assume (cf. Remark 2.8 and Proposition 5.5 below) that the intersection

$$
T:=\left(\mathbb{P}^{2} \times \mathbb{P}^{3}\right) \cap \mathbb{P}^{9}
$$

is transversal, so that $T$ is a smooth threefold with $K_{T} \sim \mathcal{O}_{T}(-1,-2)$. Hence, by the adjunction formula, $S$ is a divisor of type $(1,2)$ in $T$ and we can conclude as follows.

Lemma 5.1. The surface $S$ belongs to $\left|-K_{T}\right|$ and is a complete intersection in $\mathbb{P}^{2} \times \mathbb{P}^{3}$ of three divisors, respectively of type $(1,1),(1,1)$ and $(1,2)$.

Let $(x, y):=\left(x_{0}: x_{1}: x_{2}\right) \times\left(y_{0}: y_{1}: y_{2}: y_{3}\right)$ be coordinates on $\mathbb{P}^{2} \times \mathbb{P}^{3}$. The equations of $S$ in $\mathbb{P}^{2} \times \mathbb{P}^{3}$ can be written as

$$
a_{0} x_{0}+a_{1} x_{1}+a_{2} x_{2}=b_{0} x_{0}+b_{1} x_{1}+b_{2} x_{2}=c_{0} x_{0}+c_{1} x_{1}+c_{2} x_{2}=0,
$$


where for $i=0,1,2$ the coefficients $a_{i}$ and $b_{i}$ are linear forms while the $c_{i}$ are quadratic forms in $\left(y_{0}: y_{1}: y_{2}: y_{3}\right)$. The equations of $T$ are

$$
a_{0} x_{0}+a_{1} x_{1}+a_{2} x_{2}=b_{0} x_{0}+b_{1} x_{1}+b_{2} x_{2}=0 .
$$

The morphism $p_{T}: T \rightarrow \mathbb{P}^{3}$ is birational and its inverse is described by

$$
(y) \mapsto\left(a_{1} b_{2}-a_{2} b_{1}, a_{2} b_{0}-a_{0} b_{2}, a_{0} b_{1}-a_{1} b_{0}\right) \times\left(y_{0}: y_{1}: y_{2}: y_{3}\right) .
$$

Equivalently, $p_{T}$ is the blow-up of the scheme $\gamma$ defined by the $2 \times 2$ minors of

$$
\left(\begin{array}{ccc}
a_{0} & a_{1} & a_{2} \\
b_{0} & b_{1} & b_{2}
\end{array}\right)
$$

Since $T$ is smooth, $\gamma$ is a smooth (rational normal cubic) curve. Let $P_{\gamma}:=p_{T}^{-1}(\gamma)$ be the exceptional divisor of $p_{T}$.

Lemma 5.2. We have $P_{\gamma} \in\left|\mathcal{O}_{T}(-1,2)\right|$ and $P_{\gamma} \simeq \mathbb{P}^{1} \times \mathbb{P}^{1}$. Under this identification, $\mathcal{O}_{P_{\gamma}}(0,1) \simeq \mathcal{O}_{\mathbb{P}^{1} \times \mathbb{P}^{1}}(0,3)$ and $\mathcal{O}_{P_{\gamma}}(1,0) \simeq \mathcal{O}_{\mathbb{P}^{1} \times \mathbb{P}^{1}}(1,1)$.

Proof. We have

$$
\mathcal{O}_{T}\left(P_{\gamma}\right) \simeq \omega_{T} \otimes p_{T}^{*}\left(\omega_{\mathbb{P}^{3}}^{\vee}\right) \simeq \mathcal{O}_{T}(-1,-2) \otimes \mathcal{O}_{T}(0,4) \simeq \mathcal{O}_{T}(-1,2) .
$$

As is well known, $\mathcal{N}_{\gamma / \mathbb{P}^{3}} \simeq \mathcal{O}_{\mathbb{P}^{1}}(5) \oplus \mathcal{O}_{\mathbb{P}^{1}}(5)$, whence $P_{\gamma} \simeq \mathbb{P}^{1} \times \mathbb{P}^{1}$. Since $\gamma \subset \mathbb{P}^{3}$ is a curve of degree 3 , it follows that $\mathcal{O}_{P_{\gamma}}(0,1) \simeq \mathcal{O}_{\mathbb{P}^{1} \times \mathbb{P}^{1}}(0,3)$. Finally, we have

$$
\mathcal{O}_{\mathbb{P}^{1} \times \mathbb{P}^{1}}(-2,-2) \simeq \omega_{P_{\gamma}} \simeq \mathcal{O}_{P_{\gamma}}\left(K_{T}+P_{\gamma}\right) \simeq \mathcal{O}_{P_{\gamma}}(-2,0),
$$

whence $\mathcal{O}_{P_{\gamma}}(1,0) \simeq \mathcal{O}_{\mathbb{P}^{1} \times \mathbb{P}^{1}}(1,1)$.

Lemma 5.3. We have

$$
S \cdot P_{\gamma}=\Gamma+N_{3}+\cdots+N_{8}
$$

where $\Gamma$ is a smooth element of $\left|\mathcal{O}_{P_{\gamma}}(1,0)\right|=\left|\mathcal{O}_{\mathbb{P}^{1} \times \mathbb{P}^{1}}(1,1)\right|$. In particular, $p_{\Gamma}^{\prime}$ is a two to one map onto a line.

Moreover, $\Gamma$ has the following properties:

(i) $\Gamma \cdot N_{3}=\cdots=\Gamma \cdot N_{8}=1$ and $\Gamma \cdot N_{1}=\Gamma \cdot N_{2}=2$.

(ii) $\Gamma+N_{1}+N_{2} \sim R^{\prime}$.

Proof. We know that $N_{3}, \ldots, N_{8}$ are contracted by $p_{S}$, whence they are six disjoint fibres of $p_{P_{\gamma}}: P_{\gamma} \rightarrow \gamma$. On the other hand, $S \in\left|\mathcal{O}_{T}(1,2)\right|$, hence its restriction to $P_{\gamma}$ belongs to $\left|\mathcal{O}_{\mathbb{P}^{1} \times \mathbb{P}^{1}}(1,7)\right|$ by Lemma $\left[5.2\right.$. This implies that $\Gamma \in\left|\mathcal{O}_{\mathbb{P}^{1} \times \mathbb{P}^{1}}(1,1)\right|=\left|\mathcal{O}_{P_{\gamma}}(1,0)\right|$, and it immediately follows that $p^{\prime}$ maps $\Gamma$ two to one onto a line. If $\Gamma$ is not smooth, then it contains a fibre $N_{9}$ of $p_{P_{\gamma}}$. But then one can check (on $S$ ) that $N_{9}$ is orthogonal to $R, N_{1}, \ldots, N_{8}$. Hence Pic $S$ has rank $\geq 10$, against the generality of $S$. The properties (i) and (ii) are easy to check.

Consider the line $\ell:=p^{\prime}(\Gamma)$ and the surface

$$
P_{\ell}:=p^{-1}(\ell) \cap T \in\left|\mathcal{O}_{T}(1,0)\right| .
$$

Let $l_{0} x_{0}+l_{1} x_{1}+l_{2} x_{2}=0$ be the equation of $\ell$, with $l_{0}, l_{1}, l_{2} \in \mathbb{C}$. Then $P_{\ell}$ is defined by

$$
l_{0} x_{0}+l_{1} x_{1}+l_{2} x_{2}=a_{0} x_{0}+a_{1} x_{1}+a_{2} x_{2}=b_{0} x_{0}+b_{1} x_{1}+b_{2} x_{2}=0 .
$$


The surface $P_{\ell}$ is a $\mathbb{P}^{1}$-bundle over $\ell$ and $p\left(P_{\ell}\right) \subset \mathbb{P}^{3}$ is a quadric through $\gamma$ defined by the equation

$$
\operatorname{det}\left(\begin{array}{ccc}
l_{0} & l_{1} & l_{2} \\
a_{0} & a_{1} & a_{2} \\
b_{0} & b_{1} & b_{2}
\end{array}\right)=0
$$

Lemma 5.4. One has

$$
S \cdot P_{\ell}=\Gamma+N_{1}+N_{2}
$$

Moreover, $p\left(P_{\ell}\right)$ is smooth and $P_{\ell} \simeq \mathbb{P}^{1} \times \mathbb{P}^{1}$, with $\mathcal{O}_{P_{\ell}}(1,1) \simeq \mathcal{O}_{\mathbb{P}^{1} \times \mathbb{P}^{1}}(2,1)$.

Proof. The first assertion follows from Lemma 5.3(ii) and (19). Next assume $p\left(P_{\ell}\right)$ is singular. Then it is a rank 3 cone of vertex $e=p\left(N_{1}\right) \cap p\left(N_{2}\right)$, and $e \in \gamma$. But then the curve $p_{T}^{-1}(e)$ is contained in $S \cap P_{\ell}$ as a proper component of $\Gamma$, against the irreducibility of $\Gamma$. Finally, since $p\left(P_{\ell}\right)$ is a smooth quadric, we have $\mathcal{O}_{P_{\ell}}(0,1) \simeq \mathcal{O}_{\mathbb{P}^{1} \times \mathbb{P}^{1}}(1,1)$. Hence, the isomorphism $\mathcal{O}_{P_{\ell}}(1,1) \simeq \mathcal{O}_{\mathbb{P}^{1} \times \mathbb{P}^{1}}(2,1)$ follows.

In the considerations so far, $\gamma, T$ and $P_{\gamma}$ are fixed and independent of $S$, whereas $\Gamma$ depends on $S$ and determines the line $\ell \subset \mathbb{P}^{2}$ and thus the surface $P_{\ell}$. Actually, $\ell$ alone determines both $P_{\ell}$ and $\Gamma$, as $P_{\ell}=p_{T}^{\prime-1}(\ell)$ and $\Gamma=P_{\ell} \cap P_{\gamma}$. In order to parametrize all Nikulin surfaces we will indeed let $\ell \subset \mathbb{P}^{2}$ vary.

5.1. Rationality of $\mathcal{F}_{11}^{\mathbf{N}, n s}$. Fix any smooth rational normal cubic curve $\gamma \subset \mathbb{P}^{3}$ and let $p_{T}: T \rightarrow \mathbb{P}^{3}$ be the blow-up along $\gamma$ with exceptional divisor $P_{\gamma}$. Then $T \subset \mathbb{P}^{2} \times \mathbb{P}^{3}$ and we denote as before by $p_{T}^{\prime}: T \rightarrow \mathbb{P}^{2}$ the first projection. Any line $\ell \subset \mathbb{P}^{2}$ determines a surface $P_{\ell}:=p^{\prime-1}(\ell) \cap T \in\left|\mathcal{O}_{T}(1,0)\right|$ and a curve $\Gamma_{\ell}:=P_{\ell} \cap P_{\gamma} \in\left|\mathcal{O}_{\mathbb{P}^{1} \times \mathbb{P}^{1}}(1,1)\right|$, which is smooth for general $\ell$.

Proposition 5.5. Let $\ell$ be general. Then a general member of $\left|\mathcal{I}_{\Gamma_{\ell} / T}(1,2)\right|$ is smooth and every smooth $S \in\left|\mathcal{I}_{\Gamma_{\ell} / T}(1,2)\right|$ is a non-standard Nikulin surface of genus 11 polarized by $\mathcal{O}_{S}(1,2)\left(-\Gamma_{\ell}\right)$.

Moreover, $\operatorname{dim}\left|\mathcal{I}_{\Gamma_{\ell} / T}(1,2)\right|=12$.

Proof. Consider the exact sequences of ideal sheaves

$$
0 \longrightarrow \mathcal{I}_{P_{\gamma} / T}(1,2) \longrightarrow \mathcal{I}_{\Gamma_{\ell} / T}(1,2) \longrightarrow \mathcal{O}_{P_{\gamma}}(1,2)\left(-\Gamma_{\ell}\right) \longrightarrow 0
$$

and

$$
0 \longrightarrow \mathcal{I}_{P_{\ell} / T}(1,2) \longrightarrow \mathcal{I}_{\Gamma_{\ell} / T}(1,2) \longrightarrow \mathcal{O}_{P_{\ell}}(1,2)\left(-\Gamma_{\ell}\right) \longrightarrow 0
$$

By (19) and Lemma 5.2 we have

$$
\mathcal{I}_{P_{\gamma} / T}(1,2) \simeq \mathcal{O}_{T}(2,0) \text { and } \mathcal{I}_{P_{\ell} / T}(1,2) \simeq \mathcal{O}_{T}(0,2)
$$

and by Lemmas 5.2, 5.3 and 5.4 we have

$$
\begin{aligned}
& \mathcal{O}_{P_{\gamma}}(1,2)\left(-\Gamma_{\ell}\right) \simeq \mathcal{O}_{P_{\gamma}}(0,2) \simeq \mathcal{O}_{\mathbb{P}^{1} \times \mathbb{P}^{1}}(0,6) \\
& \mathcal{O}_{P_{\ell}}(1,2)\left(-\Gamma_{\ell}\right) \simeq \mathcal{O}_{P_{\ell}}(2,0) \simeq \mathcal{O}_{\mathbb{P}^{1} \times \mathbb{P}^{1}}(2,0) .
\end{aligned}
$$

Thus, either of (20) and (21) shows that $\mathcal{I}_{\Gamma_{\ell} / T}(1,2)$ is globally generated. In particular, a general $S \in\left|\mathcal{I}_{\Gamma_{\ell} / T}(1,2)\right|$ is smooth and hence a $K 3$ surface by adjunction. 
From (201)-(24) one obtains that $h^{0}\left(\mathcal{I}_{\Gamma_{\ell} / T}(1,2)\right)=13$ and that the restriction maps

$$
\begin{aligned}
\rho_{\gamma}:\left|\mathcal{I}_{\Gamma_{\ell} / T}(1,2)\right| \longrightarrow \Gamma_{\ell}+\left|\mathcal{O}_{P_{\gamma}}(0,2)\right| & =\Gamma_{\ell}+\left|\mathcal{O}_{\mathbb{P}^{1} \times \mathbb{P}^{1}}(0,6)\right| \\
\rho_{\ell}:\left|\mathcal{I}_{\Gamma_{\ell} / T}(1,2)\right| \longrightarrow \Gamma_{\ell}+\left|\mathcal{O}_{P_{\ell}}(2,0)\right| & =\Gamma_{\ell}+\left|\mathcal{O}_{\mathbb{P}^{1} \times \mathbb{P}^{1}}(2,0)\right|
\end{aligned}
$$

are surjective. A general member of $\left|\mathcal{O}_{P_{\gamma}}(0,2)\right|$ and of $\left|\mathcal{O}_{P_{\ell}}(2,0)\right|$ consists of 6 and 2 disjoint lines, respectively. Hence a general $S \in\left|\mathcal{I}_{\Gamma_{\ell} / T}(1,2)\right|$ contains a configuration of 8 disjoint lines, say $N_{1}, \ldots, N_{8}$, such that

(25) $\Gamma_{\ell}+N_{1}+N_{2}=S \cdot P_{\ell} \in\left|\mathcal{O}_{S}(1,0)\right|$ and $\Gamma_{\ell}+N_{3}+\cdots+N_{8}=S \cdot P_{\gamma} \in\left|\mathcal{O}_{S}(-1,2)\right|$

(using (19) and Lemma 5.2). By (25), we also get

$$
2 \Gamma_{\ell}+N_{1}+\cdots+N_{8} \in\left|\mathcal{O}_{S}(0,2)\right|,
$$

whence $N_{1}+\cdots+N_{8}$ is divisible by 2 in Pic $S$. One easily checks that

$$
\mathcal{O}_{S}(1,2)\left(-\Gamma_{\ell}\right) \sim \mathcal{O}_{S}(0,2)+N_{1}+N_{2} \sim \mathcal{O}_{S}(2,0)+N_{3}+\cdots+N_{8}
$$

is a genus 11 polarization having zero intersection with all $N_{1}, \ldots, N_{8}$. The fact that $S$ is of non-standard type is an immediate consequence of (25).

By the considerations at the beginning of the section, any smooth genus 11 Nikulin surface of nonstandard type is an element of $\left|\mathcal{O}_{T}(1,2)\right|$ and defines a smooth $\Gamma_{\ell}$ mapping $2: 1$ to a line $\ell$ on $\mathbb{P}^{2}$ under $p$. It moreover comes equipped with 6 horizontal rational curves $N_{3} \cup \cdots \cup N_{8}$, and thus determines 6 points on $\gamma$.

Lemma 5.6. Fix a general line $\ell \subset \mathbb{P}^{2}$ and six general points $p_{3}, \ldots, p_{8}$ on $\gamma$. Let $N_{i}=P_{\gamma} \cap p_{T}^{-1}\left(p_{i}\right), i=3, \ldots, 8$. Then $\operatorname{dim}\left|\mathcal{I}_{\Gamma_{\ell}+N_{3}+\cdots+N_{8} / T}(1,2)\right|=6$.

Proof. The statement follows from the ideal sequence

$$
0 \longrightarrow \mathcal{I}_{P_{\gamma} / T}(1,2) \longrightarrow \mathcal{I}_{\Gamma_{\ell}+N_{3}+\cdots+N_{8} / T}(1,2) \longrightarrow \mathcal{I}_{\Gamma_{\ell}+N_{3}+\cdots+N_{8} / P_{\gamma}}(1,2) \longrightarrow 0,
$$

along with (22) and the fact that $\mathcal{I}_{\Gamma_{\ell}+N_{3}+\cdots+N_{8} / P_{\gamma}}(1,2) \simeq \mathcal{O}_{P_{\gamma}}$ by Lemma 5.3 .

We consider the $\mathbb{P}^{6}$-bundle $\mathcal{P}$ over $\left(\mathbb{P}^{2}\right)^{\vee} \times \operatorname{Sym}^{6}(\gamma)$, whose fiber over the point $\left(\ell, p_{3}+\cdots+p_{8}\right)$ is the linear system $\left|\mathcal{I}_{\Gamma_{\ell}+N_{3}+\cdots+N_{8} / T}(1,2)\right|$ with $N_{i}=P_{\gamma} \cap p_{T}^{-1}\left(p_{i}\right)$. Our construction provides a dominant rational moduli map

$$
f: \mathcal{P}-->\mathcal{F}_{11}^{\mathbf{N}, n s},
$$

and the fibers are orbits of the group of automorphisms of $T$ that fix the exceptional divisor $P_{\gamma}$, namely, of the group of automorphisms of $\gamma \subset \mathbb{P}^{3}$. In particular $\mathcal{F}_{11}^{\mathbf{N}, n s}$ is birational to $\mathcal{P} / \operatorname{Aut}(\gamma)$.

Theorem 5.7. The moduli space $\mathcal{F}_{11}^{\mathbf{N}, n s}$ is rational.

Proof. Since there are no non-trivial automorphisms of $\mathbb{P}^{1}$ mapping a set of 6 general points to itself, $\mathcal{P} / \operatorname{Aut}(\gamma)$ is birational to a $\mathbb{P}^{6}$-bundle over $\left.\left(\mathbb{P}^{2}\right)^{\vee} \times\left(\operatorname{Sym}^{6}(\gamma)\right) / \operatorname{Aut}(\gamma)\right)$. It is then enough to recall that $\left.\operatorname{Sym}^{6}\left(\mathbb{P}^{1}\right)\right) / \operatorname{Aut}\left(\mathbb{P}^{1}\right)$ is birational to the moduli space $\mathcal{M}_{2}$ of genus 2 curves, which is known to be rational, cf. Ig. 
5.2. The fibre of the Prym-Nikulin map $\chi_{11}^{n s}$. The genus 11 case of Theorem 1.1 is a consequence of the following:

Lemma 5.8. Let $(S, M, H)$ be a general member of $\mathcal{F}_{11}^{\mathbf{N}, n s}$. For any $C \in|H|$, the linear system $\left|\mathcal{I}_{C / T}(1,2)\right|$ is a pencil of nonisomorphic non-standard Nikulin surfaces of genus 11 .

Proof. The ideal sequence of $C \subset S \subset T$ twisted by $\mathcal{O}_{T}(1,2)$ becomes

$$
0 \longrightarrow \mathcal{O}_{T} \longrightarrow \mathcal{I}_{C / T}(1,2) \longrightarrow \mathcal{O}_{S}(\Gamma) \longrightarrow 0,
$$

by Proposition 5.5. As a consequence, the 1-dimensional linear system $\left|\mathcal{I}_{C / T}(1,2)\right|$ contains $C \cup \Gamma$ as its base locus and thus parametrizes Nikulin surfaces again by Proposition 5.5. Let $S^{\prime}, S^{\prime \prime} \in\left|\mathcal{I}_{C / T}(1,2)\right|$ be two distinct points parametrizing isomorphic Nikulin surfaces. Then there exists $\alpha \in \operatorname{Aut}(T)$ such that $\alpha\left(S^{\prime}\right)=S^{\prime \prime}, \alpha(\Gamma)=\Gamma$ and $\alpha(C)=C$. In particular, such an $\alpha$ would induce a non-trivial automorphism of $C$. Note that the image of $m_{11}^{\mathbf{N}, n s}$ has dimension at least $22-1=21$, which is an upper bound for the dimension of any component of the locus in $\mathcal{M}_{11}$ of curves with a non-trivial automorphism, cf. Co. However, this bound is reached only by the hyperelliptic locus and $[C]$ does not lie in it as its Clifford index is 4 by [KLV, Prop. 2.3].

\section{REFERENCES}

[ACGH] E. Arbarello, M. Cornalba, P. Griffiths, J. D. Harris, Geometry of Algebraic Curves. Vol. I, Grundl. mathematischen Wiss. 267 (1985), Springer-Verlag New York.

[Be] A. Beauville, Variétés de Prym et jacobiennes intermédiaires, Ann. Sci. Éc. Norm. Supér. 10 (1977), 309-391.

[Co] M. Cornalba, On the locus of curves with automorphisms, Ann. Mat. Pura Appl. 149 (1987), 135-151.

[Do] I. Dolgachev, Mirror symmetry for lattice polarized K3 surfaces, J. Math. Sciences 81 (1996), 2599-2630.

[DN] J.M. Drezet, M.S. Narasimhan Groupe de Picard des variétés de modules de fibrés semi-stables sur les courbes algébriques, Invent. Math. 97(1989), 53-94.

[FK1] G. Farkas, M. Kemeny, The generic Green-Lazarsfeld secant conjecture, Invent. Math. 203 (2016), 265-301.

[FK2] G. Farkas, M. Kemeny, The Prym-Green Conjecture for torsion line bundles of high order, Duke Math. J. 166 (2017), 1103-1124.

[FV] G. Farkas, S. Verra, Moduli of theta characteristics via Nikulin surfaces, Math. Annalen 354 (2012), 465-496.

[GS] A. Garbagnati, A. Sarti, Projective models of $K 3$ surfaces with an even set, Adv. Geometry 8 (2008), 413-440.

[Ig] J. -I. Igusa, Arithmetic Variety of Moduli for Genus Two, Annals of Mathematics 72, (1960), 612-649.

[KLV] A. L. Knutsen, M. Lelli-Chiesa, A. Verra, Half Nikulin surfaces and moduli of Prym curves, arXiv:1708.07339 (2017).

[LC] M. Lelli-Chiesa, Generalized Lazarsfeld-Mukai bundles and a conjecture of Donagi and Morrison, with an Appendix joint with A. L. Knutsen, Adv. Math. 268 (2015), 529-563.

[Mu] D. Mumford, Varieties Defined by Quadratic Equations. In: Questions on Algebraic Varieties. C.I.M.E. Summer Schools, vol 51. Springer, Berlin, Heidelberg.

[Ra] C. P. Ramanujam, Remarks on the Kodaira vanishing theorem, J. Indian Math. Soc. (N.S.) 1 (1972), 121-124.

[Re] M. Reid, Hyperelliptic linear systems on a KS surface, J. Lond. Math. Soc. 13 (1976) 427-437.

[SD] B. Saint-Donat, Projective models of K3 surfaces, Amer. J. Math. 96 (1974), 602-639.

[Te] M. Teixidor i Bigas, Half-canonical series on algebraic curves, Trans. Amer. Math. Soc. 302 (1987), 99-115. 
[vGS] B. van Geemen, A. Sarti, Nikulin involutions on K3 surfaces, Math. Z. 255 (2007), 731-753.

A. L. Knutsen, Department of Mathematics, University of Bergen, Postboks 7800, 5020 Bergen, Norway

E-mail address: andreas.knutsen@math.uib.no

M. Lelli-Chiesa, Dipartimento di Ingegneria e Scienze dell'Informazione e Matematica, Università degli Studi dell'Aquila, Via Vetoio, località Coppito, 67100 L'Aquila, Italy E-mail address: margherita.lellichiesa@univaq.it

A. Verra, Dipartimento di Matematica, Università Roma Tre, Largo San Leonardo Murialdo, 00146 Roma, Italy

E-mail address: verra@mat.uniroma3.it 